\title{
$5139 \mathrm{~s}$
}

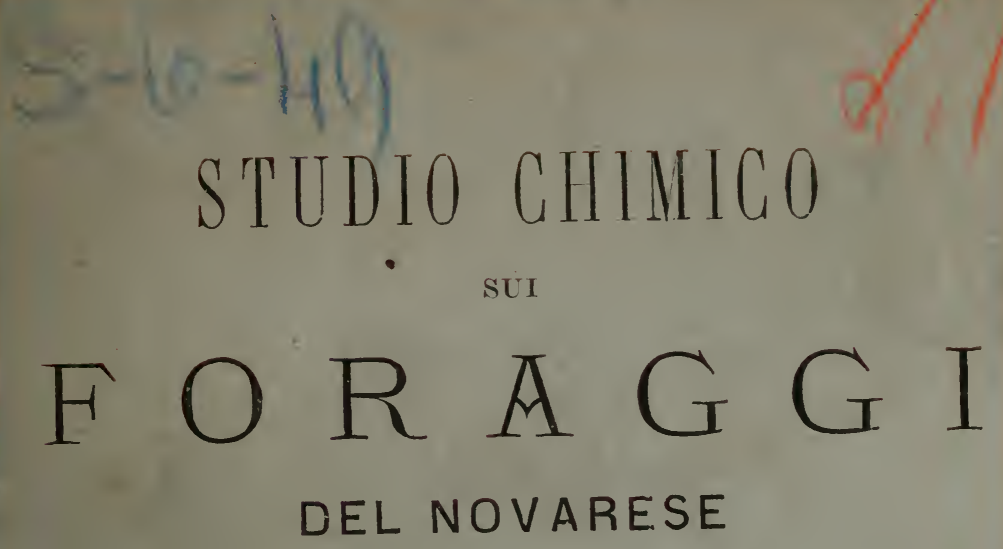

DI

\section{LEOPOLDO SILLESTRINI}

\author{
PROFESSORE DI CHIMICA GENERALE ED AGRARIA \\ NELL' ISTITUTO 'TECNICO \\ DI NOVARA.
}

Pubblicazione fatta col concorso del Comizio Agrario.

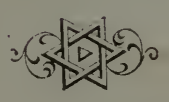

NOVARA

TIPOGRAFIA DELLA RIVISTA DI CONTABILITÀ

1881 . 


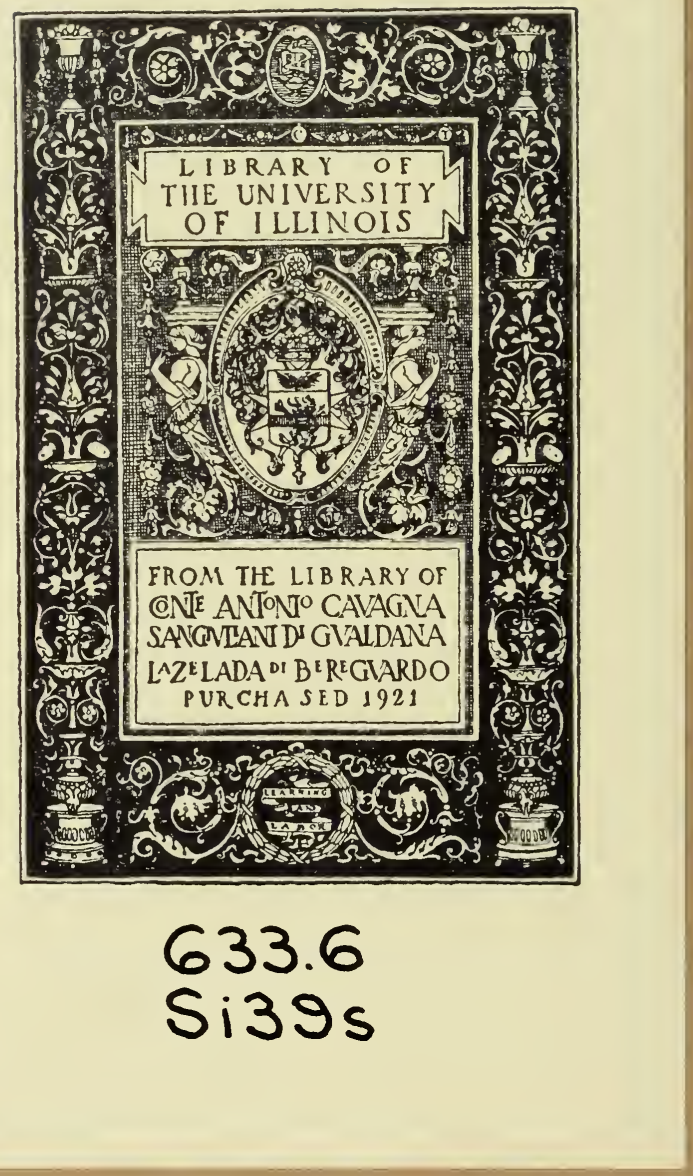




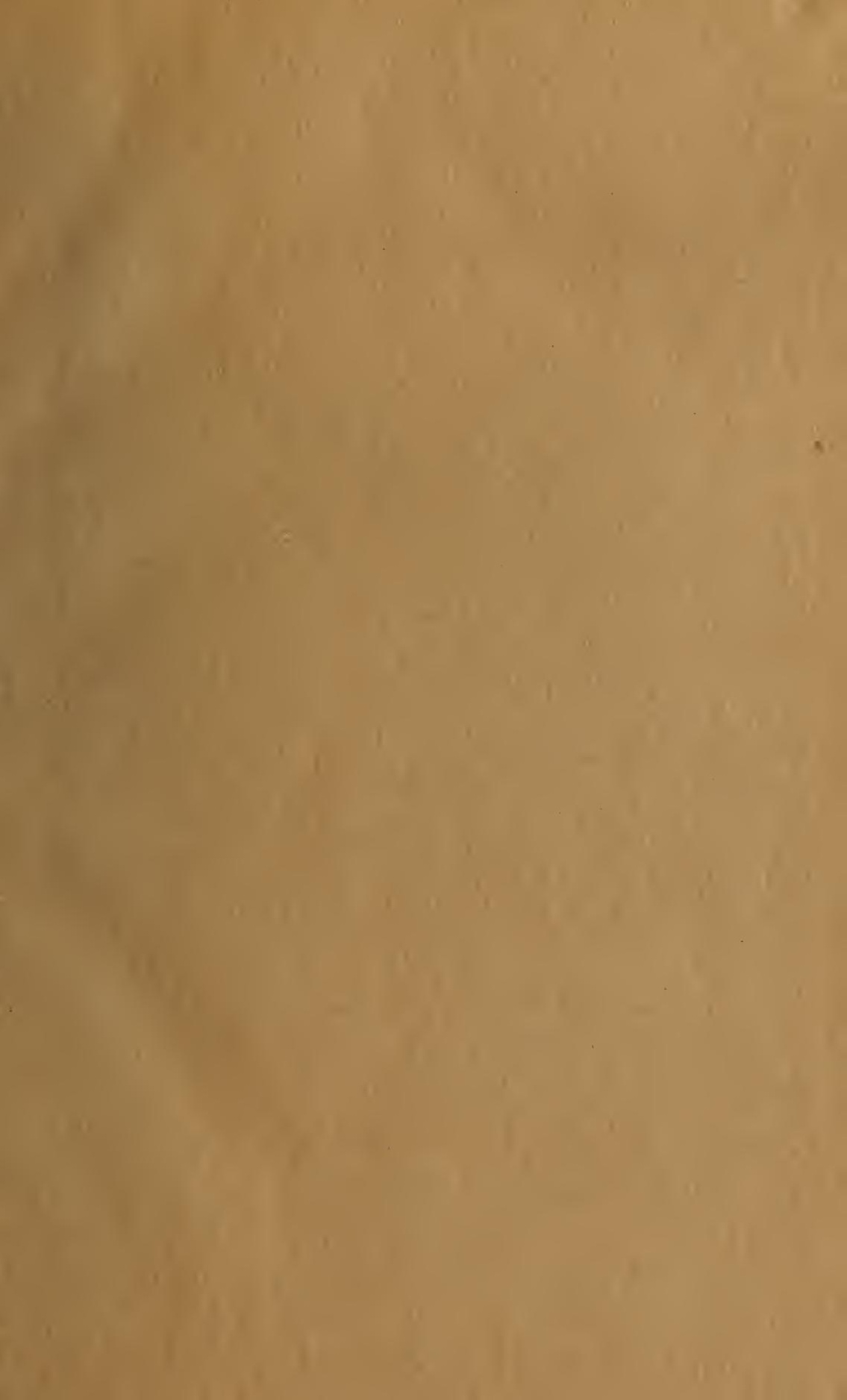



. 
Digitized by the Internet Archive in 2012 with funding from

University of Illinois Urbana-Champaign 


\section{STUDIO CHIMICO}

SUI

$\mathrm{F} O \mathrm{R}$ A G G I DEL NOVARESE

DI

\section{LEOPOLDO SILVESTRINI}

PROFESSORE DI CHIMICA GENERALE ED AGRARIA

\section{NELL' ISTITUTO 'TECNICO}

DI NOVARA.

Pubblicazione fatta col concorso del Comizio Agrario.

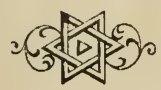

ITOVARA

TIPOGRAFIA DELLA RIVISTA DI CONTABILITÀ

1881. 

ALL'ONOREVOLE PRESIDENTE

DEL COMIZIO A GRARIO

DI NOVARA

\section{CAV. LUIGI GOTTARDO PRINA}

QUESTO LAVORO OFFRE RIVERENTE

\section{LEOPOLDO SILVESTRINI}

IN TESTIMONIANZA DI STIMA. 

La composizione chimica dei foraggi che vengono all' uopo impiegati, concorre pure fortemente a rendere piú complicato il problema dell'alimentazione del bestiame, modificando la stabilita proporzione fra il peso vivo degli animali e la quantita del foraggio ad essi occorrente; infatti i vari foraggi variano nella loro composizione chimica, nella proporzione cioé ed anco, qualche volta, nella natura dei loro materiali immediati detti plastici e respiratori che li costituiscano, e ciò secondo le, condizioni climatologiche della regione nella quale vegetano, secondo la chimica composizione dei terreni e le proprietà fisiche loro.

Queste ultime, in particolar modo, sono le ragioni per le quali lo scrivente fino dall'anno 1880 si è occupato del presente lavoro, ben sapendo come debba sommamente interessare agli agricoltori Novaresi, la conoscenza della chimica composizione dei foraggı che impiegano per lo allevamento del loro bestiame.

Variando, come più sopra si è detto, la composizione chimica dei foraggi, vario pure deve essere il loro potere nutritivo; da ciò la quasi assoluta necessità per l'agricoltore di conoscere la chimica composizione dei suoi foraggi, onde poter ricavare nell'allevamento del bestiame il maggior vantaggio possibile, inquantochè sebbene diversi sono i bisogni di alimento negli animali secondo la specie, l'età, la natura dei prodotti che debbono fornire, ecc, ecc, debbono sempre ricevere col foraggio, in quantità conveniente, tutte quelle sostanze che sono necessarie per la loro nutrizione completa, dal punto di vista dello. scopo pel quale vengano allevati.

I materiali che costituiscono i foraggi e che gli animali utilizzano per la loro nutrizione sono in parte di natura organica, e questi sono i più abbondanti, ed in parte di natura minerale. I prımi possonsi dividere in materiali azotati, detti proteici ed anco plastici ed in materiali ternari non azotati, detti respiratori.

I piú importanti fra i materiali organici certamente sono gli azotati o proteici, siccome quelli che nell'organismo animale si cangiano in nervi, sangue, muscoli, ecc., e presiedono alla formazione delle materie grasse.

Nei foraggi questi principii essenzialissimi, per dire il vero generalmente si trovano in piccola quantità; hanno del resto 
una grande analogia con i corpi albuminoidi degli animali (albumina, fibrina, caseina, ecc.) la stessa compjosizione chimica, e nei vegetali si trovano tanto allo stato solido (legumina, glutine) che allo stato liquido (albumina).

La proporzione di questi preziosi materiali varia nelle piante secondo la specie, secondo la natura chimica del terreno sul quale le piante vivono, secondo la natura dei concimi impiegati. secondo le condizioni di clima, di temperatura, ecc: nelle quali le piante si sono trovate nel tempo della loro vita; e si pui ancora asserire che nei vegetali tanto più abbondano le sostanze proteiche quanto piú sono giovani e quanto più il terreno sul quale vegetano si trova ricco di composti ammoniacali o di nitrati, composti questi che col loro azoto disponibile proviocano, nelle cellule delle piante, una più abbondante produzione di principii proteici.

Le sostanze idrocarbonate (materiali ternari detti ancora respiratori) sono, come le azotate, indispensabili alla vita animale, infatti sono queste che concorrono ad intrattenere la respirazione, a produrre e mantenere il calore animale, e ad ingenerare le materie grasse. Questi principii si incontrano piuttosto in abbondanza nelle piante e costituiscono una classe piuttosto numerosa di composti immediati i piú importanti dei quali sono l'amido, lo zucchero, il glucosio, la destrina, le materie grasse le gomme, le resine e la cellulosa o tessuto cellulare delle piante, composto pure questo ternario della classe dei veri idrati di carbonio.

Fra tutte queste sostanze quelle che più di tutte coucorrono alla nutrizione degli animali, perchè facilmente digerite e trasformate, sono l'amido e gli zuccheri.

Le materie grasse pure concorrono alla produzione del calore animale, alla formazione dei grassi animali e favoriscono la digestibilità delle altre sostanze; nelle piante però, eccettuati i semi detti oleosi, si trovano sempre in piccolissima proporzione.

La cellulosa che è quella sostanza che costituisce i tessuti dei vegetali e ne forma per cosi dire lo scheletro, fu considerata per molto tempo come affatto inutile perchè non assimilabile, in oggi peró é noto per fatti sperimentalmente dimostrati che $\mathrm{i}$ ruminanti sono capaci di assimilarne dalle 40 alle 60 parti su 
100 contenute nella razione, specialmente quando in questa scarseggiano le altre sostanze nutritive.

Nelle piante questo composto immediato si trova in abbondanza ma in stati differenti, secondo la loro specie e la loro età; da cio ben si comprende come nel foraggio questo composto si trovi in uno stato tanto più facilmente digeribile, quanto più giovani sono le piante erbacee che lo costituiscono.

I componenti minerali pure sono della massima necessitả per lo sviluppo dell'organismo animale, e nei foraggi vi si trovano sempre in proporzione superiore al bisogno.

Premesse queste generalità, credo ora opportuno di esporre in modo sommario il procedimento tenuto nelle varie ricerche e valutazioni chimiche, praticate allo scopo di determinare quantitativamente la immediata composizione dei piú importanti foraggi del Novarese ed il loro potere nutritivo.

Tutti i campioni sottoposti all'analisi furono raccolti e portati in laboratorio allo stato fresco appena falciati, quindi vennero disseccati al sole nel modo e con le norme che generalmente vengono poste in pratica dagli agricoltori.

I foraggi, dei quali si riporta la composizione allo stato di erba, vennero dapprima in proporzione rigorosamente determinata, tenuti alla stufa riscaldata $a+100$ fino a peso costante per la determinazione dell'acqua, quindi in parte diligentemente polverizzati onde i risultati dell'analisi ritraessero fedelmente la vera composizione del foraggio, ed in parte ridotti in cenere bianca, e questa serbata in vaso ermeticamente chiuso.

Dai campioni vari di erba medica e di trifoglio vennero tolte accuratamente le foglie e quindi dopo di avere con esattezza determinata l'acqua contenuta in queste e nei rispettivi steli, vennero sempre separatamente disseccati alla stufa, in parte polverizzati ed in parte ridotti in cenere bianca.

Una seconda porzione di detti foraggi (foglie e steli di erba medica e di trifoglio) venne pure disseccata al sole e ridotta in foraggio secco, e questo conservato in vasi chiusi per l'analisi.

Tale operazione si credette opportuno di praticare, onde sperimentalmente dimostrare agli agricoltori come nei mangimi surricordati le sostanze nutritive abbondino nelle foglie e scarseggino per converso negli steli, e come si esponga ad una grave 
perdita nel loro valore nutritivo, quell'agricoltore che per poca diligenza nel confezionamento dei medesimi, lasciasse disperdere una parte delle foglie che sole in alto grado sono provvedute dei materiali necessari all'alimentazione del bestiame.

Come ho riferito più sopra, l'acqua in tutti i mangimi venne determinata mantenendo alla stufa $a+100$ fino a peso costante una data quantità di foraggio verde o disseccato al sole.

La materia grassa si ottenne fortemente imbruttata di clorofilla e materie resinose, trattando in un piccolo apparecchio da spostamento a processo continuo, un dato peso di foraggio in polvere secca, e la soluzione eterea evaporando quindi in capsula di vetro rigorosamente tarata.

Le materie proteiche vennero determinate valutando lo azoto contenuto in un peso noto di foraggio, col procedimento di Will e Warentrapp, ricordando che parti 15,5 di azoto corrispondono a parti 100 di materiali albuminosi.

La cellulosa venne isolata ed esattamente pesata, mantenendo in digestione per varie ore a +70 ' nell'acido solforico diluito del suo peso d'acqua un dato peso di foraggio in polvere, onde disciogliere le materie azotate e trasformare l'amido in destrina e glucosio.

Il liquido fortemente acido, dopo opportuna diluzione con acqua, venne gettato su di un filtro pesato, e la cellulosa rimasta sul medesimo lavata ripetutamente dapprima con acqua calda, poi con una diluita soluzione di idrato potassico, quindi con acqua leggermente acida di acido acetıco, indi con alcool e finalmente disseccata alla stufa e pesata.

Le ceneri vennero dosate mediante la incinerazione completa in crogiuolo di platino di un noto peso di foraggio.

Finalmente, le sostanze ternarie (amido, zucchero, gomma, ecc, ecc.) vennero calcolate per differenza.

Le piante erbacee che crescono nei prati e nelle marcite del Novarese e che costituiscono i vari foraggi, appartengono ad un numero grandissimo di famiglie, ed assai difficile cosa sarebbe per chi non è cultore della botanica di rigorosamente classificarle. Tuttavia prima di praticare l'analisi chimica ho creduto opportuno di eseguire un saggio botanico sopra i molteplici campioni di foraggi freschi che furono portati in laboratorio, onde avere 
pure un dato sulla qualita delle piante che costituivano i fieni da analizzare, e la media dei risultati ottenuti si è quella rappresentata dal seguente specchietto:

Parti 100

di erba di marcita constano

di graminacee p. 53,7

»leguminose 》 18,0

" campanulacee 》 0,8

» cariofillee 》

》 composte 》

» crucifere 》

》 ombrellifere 》

»rannucolacee 》

》rosacee

» liliacee

》 chenopodiacee

- ecc, ecc.
Parti 100 di erba
di prato stabile constano

p. 46,3

》 29,7

》 1,3

\ 2,4

》 8,2

》 2,4

》 1,0

》 4,2

» 5,5

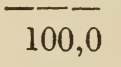

Parti 100 di erba di prato irriguo constano

p. 48,0

》 30,8

» 1,0

》 1,2

》 9,7

» 1,6

》 1,0

» 3,6

» 3,1

100,0

Ed ecco ora i resultati ottenuti dall'analisi chimica praticata sull'erba di $1^{\circ}$ taglio di N. 6 marcite del Novarese. 


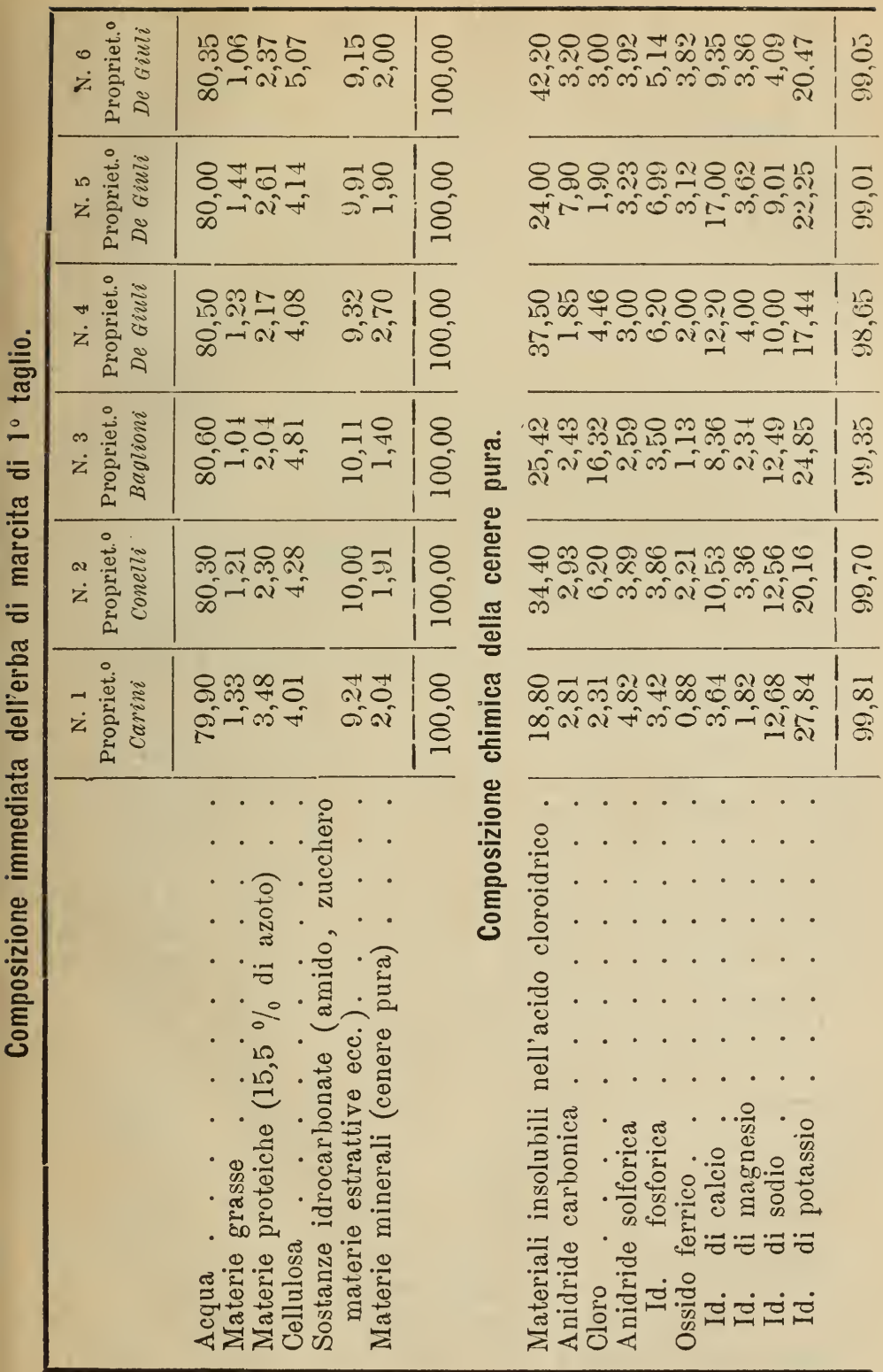


Per farsi un giusto criterio del relativo valore nutritivo di questa specialissima qualitá di foraggio fresco, bisognerebbe avere $\mathrm{i}$ resultati di altre analisi chimiche, praticate da altri sperimentatori in epoche e luoghi diversi; allora si potrebbe istituire un paragone tra $\mathrm{i}$ dati delle presenti analisi e quelli ottenuti da altri chimici, e si giungerebbe cosi a farsi un'idea ben netta sulla qualità dei foraggi analizzati. Disgraziatamente nulla ho potuto trovare in proposito, e mentre in tutti i trattati di agronomia si rinvengono dei quadri analitici osprimenti la composizione chimica del fieno maggengo e del guaime, nessun dato o quadro analitico ho potuto rinvenire che riguardi la chimica composizione dell'erba di marcita. Tuttavia, se si ispeziona il quadro surriportato, che rappresenta la composizione chimica immediata di sei campioni di erba di marcita falciata il giorno 28 marzo 1880, ben si vede come in essa abbondino piuttosto le materie grasse; infatti la quantità media di queste sostanze, riscontrata da molti sperimentatori nell'erba di prato, non è superiore di 0,8 per $\%$, mentre nell'erba delle nostre marcite raggiunge la proporzione massima di 1,44 per $\%$ e non discende che a 1,04 per $\%$.

Le sostanze proteiche nell'erba di marcita, meno che nel primo campione, sono in proporzione alquanto inferiore di quella ammessa come media nell'erba di prato. Infatti il Gohren nel suo bellissimo trattato sulle leggi naturali dell' alimentazione degli animali domestici, porta la quantità media delle sostanze proteiche nell'erba di prato a 3,1 per cento, mentre nei sei campioni di erba di marcita analizzata, meno un solo, la proporzione di queste sostanze non raggiunge il 3 per $\%$.

Le sostanze ternarie idrocarbonate per converso vi si rinvengono in giusta proporzione; infatti la media ammessa anco dal Gohren è di 10 per $\%$, e in due dei nostri campioni di erba di marcita vi si trovano nella proporzione superiore del 10 per $\%$, e negli altri quattro superiore al 9 per $\%$.

La cellulosa che dal punto di vista del potere nutritivo nei foraggi presenta la minore importanza, assolutamente scarseggia nell'erba di marcita, non raggiungendo la proporzione del 5 per \%. Ciò dipende certamente dall'età dell'erba stessa, dall'essere stata falciata cioè nella stagione ancora fredda, e 
quando l'erba non aveva ancora raggiunto il suo completo sviluppo. Infatti se si esaminano i quadri che qui in appresso vengono riportati, e che rappresentano la chimica composicione dell'erba di marcita di II. ${ }^{\circ}$, III. ${ }^{\circ}$ e IV..$^{\circ}$ taglio, ben si vede come si trovi in essa un aumento della cellulosa, mentre vi si riscontra una sensibile diminuzione nelle sostanze proteiche. Le materie grasse per converso e quelle idrocarbonate rimangono, in questi tagli, in proporzione presso a poco sempre costanti. 


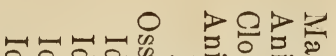

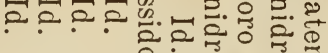
క气 유

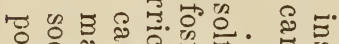

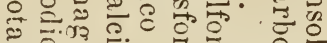

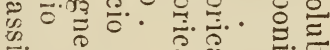
…

$8 \mid 00000-1000000$

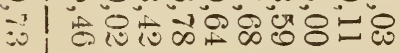

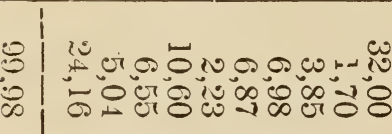

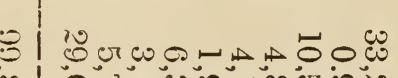
क 18000010

$5150,-100000 \omega$ 舟

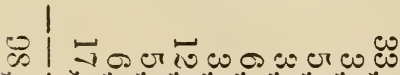

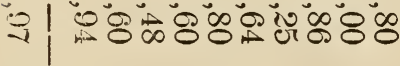

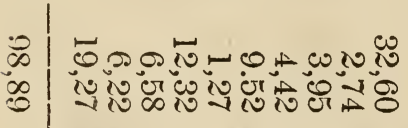

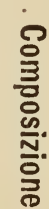

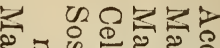

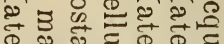

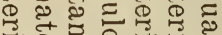
त. В बे एव

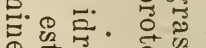
동

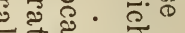
ㄷ.

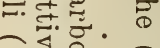
20 \& ० क क 를. 응. Е - N

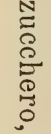




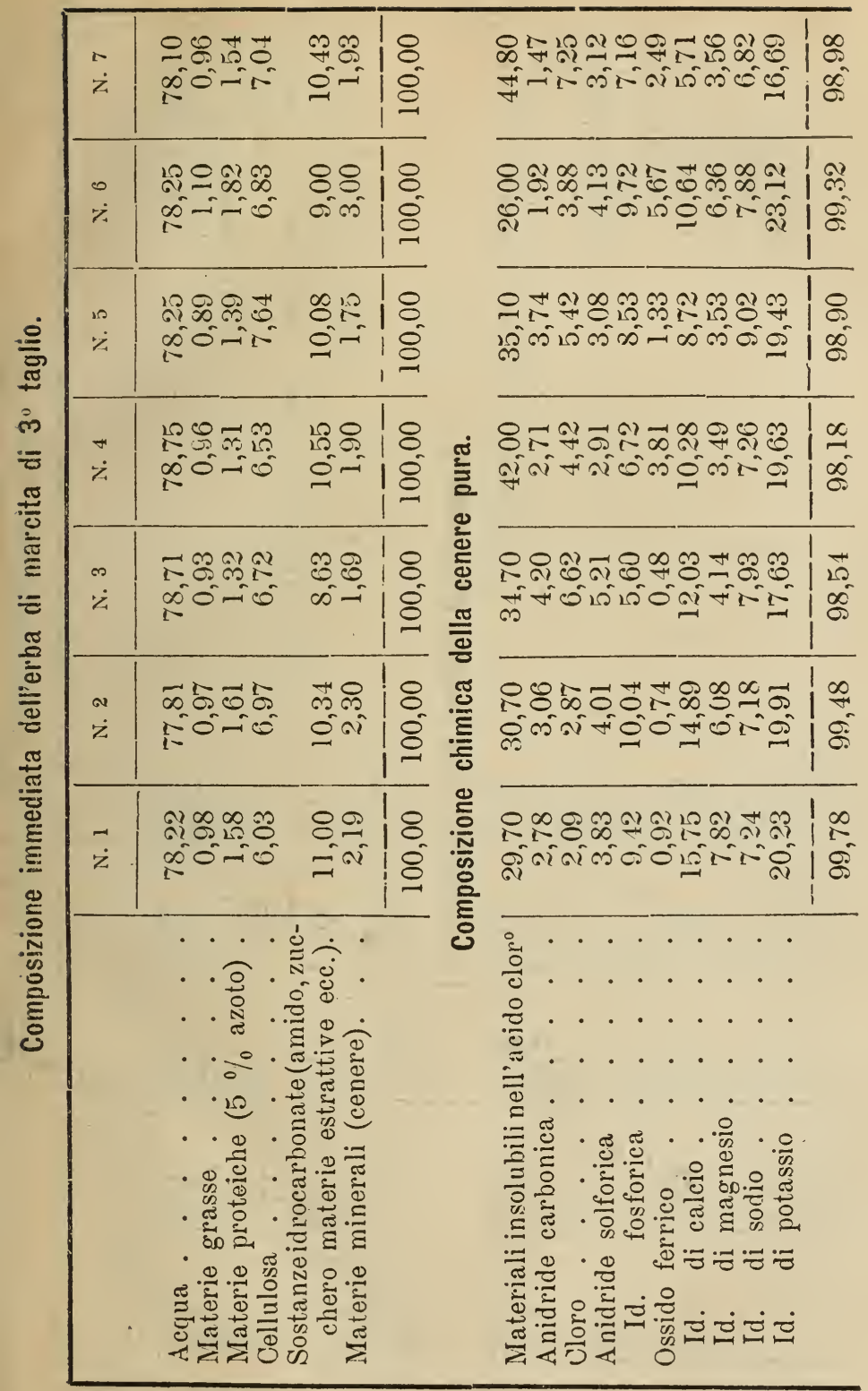




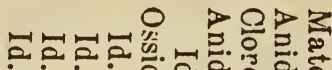

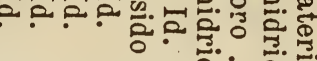

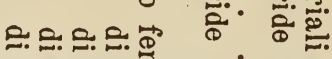
둥

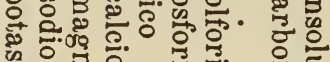

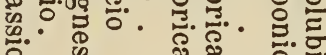
$\therefore$. . . ?

$\ldots \ldots \ldots$

. . . . . . .

……

$\ldots \ldots \ldots$.

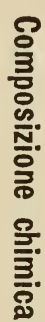

\begin{tabular}{l|l}
\hline & \\
$\infty$ & Der.
\end{tabular} 흘

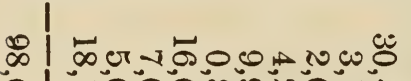
๑

\& thoovionosw o|

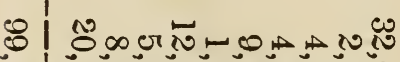

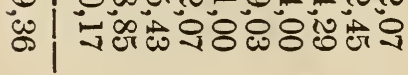
옹

of erpotonour \&

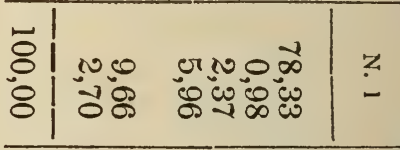

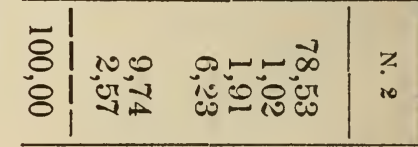
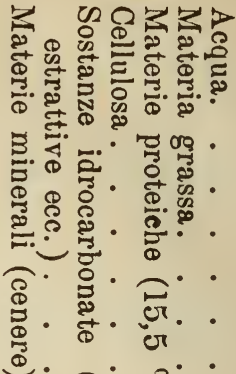

ब. $\widehat{D}^{\circ} 0^{\circ}$
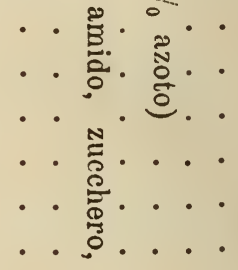

…………

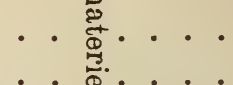

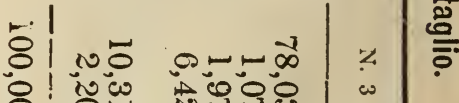

8 \%

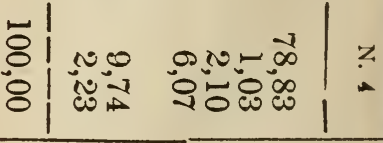

क्षे $810 \%$ 
Per quanto poi riguarda i materiali inorganici che fanno parte della composizione chimica dell'erba delle nostre marcite, $\dot{e}$ da dire che questi abbondano anzichenó, raggiungendo la media del 2 per cento di foraggio fresco; e se si vuol tener conto ancora dei vari componenti delle ceneri stesse, si vede che a paragone della cenere di fieno di prato naturale, la composizione media della quale Wolff rappresenta con le cifre seguenti :

\begin{tabular}{|c|c|c|}
\hline Anidride & silicica & 29,6 \\
\hline$\gg$ & solforica & 5,1 \\
\hline » & fosforica & 6,2 \\
\hline Calce & 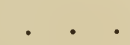 & 11,6 \\
\hline Magnesia & . & 4,9 \\
\hline Soda & . & 7,0 \\
\hline Potassa. & . & 25,6 \\
\hline
\end{tabular}

quella di erba di marcita non presenta una sensibile diversitá che nella proposizione della silice, la quale nella cenere dell'erba delle marcite Novaresi è in generale superiore di quella indicata dal Wolff come media.

La potassa invece si trova in proporzione di poco inferiore, mentre la soda in alcuni campioni di tutti e quattro i tagli trovasi in eccedenza. Se poi d'altra parte si prende a considerare la proporzione complessiva degli alcali, patentemente si vede che in vari campioni di $1 .^{\circ}, 2 .^{\circ}, 3 .^{\circ}$ e $40^{\circ}$ taglio, si trovano in quantitá minore della media indicata per le ceneri di prato stabile naturale. Ció probabilmente dipende dalle eccezionali condizioni di coltura e di vegetazione dell'erba di marcita e non dalla qualità dei terreni Novaresi, che tutti sanno essere per loro natura ricchi di silicati alcalini.

Fra i principi minerali delle ceneri delle piante da foraggio, l'anidride fosforica indubbiamente è uno dei più importanti dal punto di vista della nutrizione animale; sono infatti i fosfati terrosi che costituiscono la base delle ossa ed in particolar modo il fosfato di calce. È adunque d'uopo che di questo materiale vi sia piuttosto abbondanza che difetto nella razione alimentare; ed è certo, che se venisse fornito per qualche tempo ad un animale, un alimento mancante di una sufficicnte 
proporzione di acido fosforico e di calce, ben presto si vedrebbe deperire, e la morte non si farebbe aspettare a lungo, se tale sistema di alimentazione venisse continuato.

Or bene, guardando i quadri d'analisi più sopra tracciati, si vede che questo composto minerale scarseggia nei primi tre campioni di erba di marcita di primo taglio, ma si trova in giusta proporzione negli altri tre campioni pure di primo taglio ed abbonda in quelli di $2 .^{\circ}, 3 .^{\circ}$ e $4 .^{\circ}$ taglic.

\section{Il fiens) llei prati asciutti e dei prati irrigui.}

Prima di riportare i quadri d'analisi rappresentanti la chimica composizione del fieno maggengo e del guaime dei prati asciutti e dei prati irrigui del Novarese, credo opportuno di spendere qualche parola sui fieni in generale e sulle piante erbacee che li costituiscono.

Sensibilissime sono le differenze che si riscontrano spesso tra fieno e fieno, e pur troppo raro è il caso che si tenga conio di questo importantissimo fatto nella composizione delle razioni. Non è perció da meravigliarsi se nell'apprezzamento del valore nutritivo del fieno si hanno opinioni disparate, risultati diversi ed anco contradditori, e ciò perchè siffatto valore non solo dipende dalla natura delle piante che costituiscono il fieno, ma ancora dal modo di sua preparazione e di sua conservazione.

Secondo Grouven le erbe dei prati che costituiscono il fieno possono essere classificate, riguardo alla loro bontà, nel modo e nell'ordine seguente:

EbBe di ottima QUALItÀ

Loiessa - Lolium italicum.

Codolina - Phleum pratense.

Gramigna delle vie - Poa annua.

Gramigna canarola - Cinosurus cristatus. 
Forasacco peloso - Bromus mollis.

Erba mazzolina - Dactylis glomerata.

Orzo dei prati - Hordeum pritense.

Coda di volpe - Alopecorus pratensis.

Saggina canarola - Arrhenaterum avenaceum.

Gioglierella - Lolium perenne.

\section{Erbe DI MEDiocre QUALITȦ}

Gramigna setarola - Festuca duriuscula.

Avena pelosa - Avena pubescens.

Gramigna odorosa - Anthoxantum odoratum.

Gramigna dei prati - Poa pratensis.

Segala selvatica - Holcus lanatus.

Spannocchina - Poa trivialis.

Gramigna bianca - Avena flavesecus.

Tremolino - Briza media.

Pessime per converso, sono le erbe acide che crescono nei luoghi umidi e sortumosi, ed in particolar modo le giuncacee e le ciperacee che possono anco riescire dannose agli animali.

Il fieno di prateria naturale, dal Wolf viene distinto in tre specie. La prima comprende il migliore che $\dot{\theta}$ quello, secondo il surramentato autore, ricco di foglie e specialmente quello dei monti dal quale gli animali assimilano il 60 per $\%$ dei principii organici che contiene. Alia seconda specie riporta il fieno di bontà media il quale viene fornito dai prati irrigui; siffatto foraggio è generalmente costituito da erbe di alto caule, da erbe fine e da piccola quantitá di erbe a larghe foglie. Gli animali che se ne cibano, digeriscono il 50 e 55 per cento delle sostanze organiche che lo costituiscono. Alla terza specie finalmente riporta il fieno di cattiva qualità, quale si trae dai prati sortumosi; consta questo di erbe cresciute assai in lunghezza, grossolane ed a larghe foglie. La, digeribilità dei principii organici di questa specie non raggiunge che il 40 per $\%$. Sempre secondo il Wolf, il fieno dei prati umidi, mal- 
grado il suo rigoglioso sviluppo, é meno nutritivo di quello dei buoni prati asciutti.

Il fieno, in seguito a ripetute pioggie subisce una sensibile modificazione nella sua chimica composizione, perdendo una certa quantitá di principi nutritivi. Si veggano infatti le seguenti analisi di Stöchard e Ritthausen praticate sul fieno buono, e sul fieno colto dalla pioggia.

\section{BuON FIENO}

Fieno colto dalla pioggia

$\begin{array}{lrr}\text { Sostanze proteiche } & 7,8 & 6,5 \\ \text { Zucchero } & 0,71 & 0,12 \\ \text { Materie idrocarbonate } & 53,3 & 49,7 \\ \text { Cellulosa } & 32,1 & 36,5 \\ \text { Ceneri } & 6,1 & 7,2 \\ & -100,01 & -100,02\end{array}$

Finalmente, se il fieno viene troppo a lungo conservato perde del suo valore nutritivo; infatti il fieno troppo vecchio non ha più il suo culore, il suo odore ed il suo sapore, si rompe facilmente, e le parti piú fine e nutritive cadono in polvere e vanno quindi assolutamente perdute.

Per quanto poi riguarda il fieno di secondo taglio o guaime dal punto di vista del suo valore nutritivo, è da dire che le opinioni sono ancora molte disparate. Nella Germania meridionale per esempio e nella Svizzera, è il fieno agostano che maggiormente viene stimato, nella settentrionale per converso si preferisce il maggengo; ma cio dipende certamente dal tempo. dominante durante la sua preparazione, e in particolar modo. dalla specie delle piante erbacee che lo costituiscono.

Del resto si veggano le analisi del fieno maggengo e del guaime eseguite nella stazione di Weende, molto interessanti dal lato pratico, inquantochè dimostrano la superiorità del guaime ben preparato, sul fieno maggengo di media qualità.

100 Kilogrammi di guaime privato d'acqua contenevano in tutto 
Sostanze

organiche

Kg. 90,8
Albumina Cellulosa

16,1

23,0

Grasso

3,1

idrocarbonate

48,6

di queste sono digeribili:
56,8
10,9
15,6
1,0
36,0

100 Chilogrammi di fieno maggengo privato d'acqua contenevano in tutto

$\begin{array}{ccccc}\begin{array}{c}\text { Sostanze } \\ \text { organiche }\end{array} & \text { Albumina } & \text { Cellulosa } & \text { Grasso } & \begin{array}{c}\text { Sostanze } \\ \text { idrocarbonate }\end{array} \\ \mathrm{Kg} .93,1 & 10,9 & 29,1 & 28,8 & 50,4\end{array}$

di queste sono digeribili :

$56,8 \quad 6,0 \quad 17,0 \quad 1,0 \quad 32,5$

Ecco ora quanto scrive il Wolf sulle digeribilità dei fieni in generale :

Moltissime ricerche eseguite sul fieno pratense di diversi tagli e sulle erbe dei prati, dimostrano in modo incontestabile che quando abbonda la quantità di azoto, difetta in corrispondenza la quantità di fibra legnosa, cresce la proporzione della cenere e delle materie grasse, e rimane quasi stazionaria la quantitá delle sostanze estrattive non azotate. Col crescere della quantità dei materiali proteici in un foraggio verde o secco, si esalta in corrispondenza la digeribilitá del foraggio stesso. $\dot{E}$ poi un fatto caratteristico dei foraggi rappresentati da piante graminacee, che coll'aumentarsi della digeribilita dei corpi proteici, si aumenta pure quella delle sostanze estrattive non azotate: fatto che non si mostra cosi chiaramente e regolarmente riguardo la cellulosa e le sostanze grasse. La digeribilitá della fibra legnosa è relativamente grande in tutte le specie di fieno di prato naturale, quella del grasso per converso, è relativamente piccola.

La quantità di azoto del fieno pratense, e quella della cellulosa, porgono un criterio sufficientemente giusto per apprezzare la digeribilità ed il potere nutritivo di questo mangime. I fieni ricchi di azoto e scarsi di fibra legnosa sono i migliori, quelli cioè di piú facile digestione, hanno invece debole valore nutritivo quei fieni che sono scarsi di azoto ma ricchi di fibra legnosa. 
Le qualità di fieno scarse di azoto e di fibra legnosa, contengono quasi sempre le sostanze proteiche in forma difficilmente digeribile, e le sostanze estrattive non azotate in forma facilmente digeribile Finalmente, quelle specie di fieno che abbondano di azoto e di cellulosa, si comportano inversamente od hanno coefficienti di assimilazione medii.

La quantità di proteina del fieno naturale di primo taglio può variare dal 7 al $20 \%$ della sostanza secca, e la digeribilitá di questa proteina puó pure variare fra 39 e $70 \%$ della quantità totale. Da ció la possibilitá della presenza di una quantitá di albumina digeribile 5 volte maggiore e minore. Se poi si pensa che questo principio azotato è quello che governa l'effetto nutritivo in ogni regime di produzione, non farà più meraviglia che nella pratica si sieno ottenuti effetti molto diversi coll'impiego di diversa qualità di maggengo.

Il fieno di secondo taglio dei prati permanenti, purchè confezionato con un tempo favorevole è da considerarsi, per la composizione e la facile sua digeribilitá, come una delle migliori specie di fieno di primo taglio, dalle quali si distingue soltanto per una minore sapidità e odore aromatico. Il suo valore $\dot{e}$ peró interamente subordinato al tempo che si ha durante la sua confezione. E da ricordare infatti che l'agostano risente più del maggengo l'influenza delle cause deterioranti, perchè racchiude una maggiore dose di principii facilmente solubili, ed a motivo del suo stato fino e delicato è piú facilmente imbevuto d'acqua; dissecca poi con piú difficoltà, e entra con grande facilità in fermentazione e putrefazione. Non è dunque da meravigliarsi se questa specie di foraggio spesso si corrompe in tutta la sua massa, si copre di muffe, e ripugni in questo stato all'animale, mentre confezionato con tempo favorevole sia un eccellente mangime.

Premesse le suesposte generali considerazioni sulla bontá delle erbe dei prati naturali e sulla qualità dei fieni che esse forniscono, ecco ora nei quadri qui in appresso tracciati, la composizione chimica immediata di undici campioni di fieno maggengo di prato stabile asciutto del Novarese, di sette campioni di fieno agostano e di otto campioni di fieno di terzo taglio. 


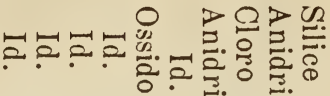

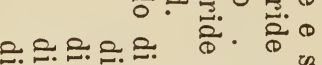
भाष ㄴำ ○得骂

- $\frac{0}{\circ} \cdot \frac{\Phi}{\circ} \cdot$.

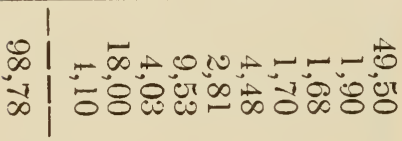

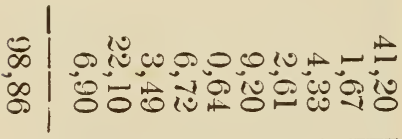

$\infty \mid$ oळe \& 1 ن

- 1 1 ○ س

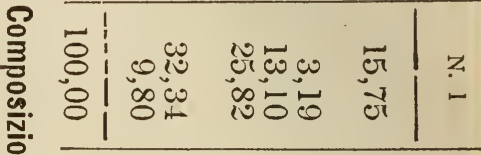

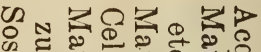
से क्षेत्र

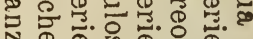

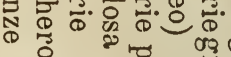
น B. 2.3 .50 इं के के के ๑ क

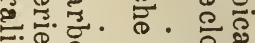
을 응 ลิ इे एँ. है. (1)

- 2 ह.

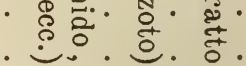

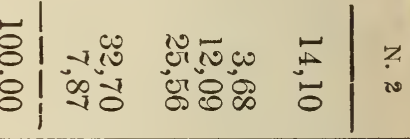

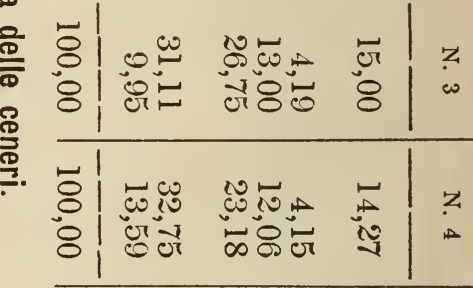

81 ง I)

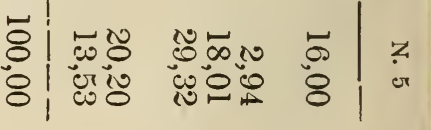

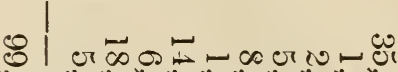
类

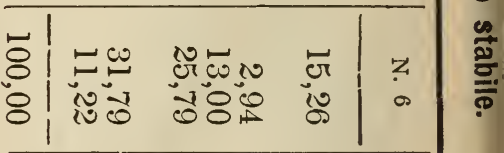

๑) ठ

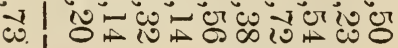

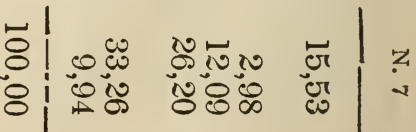




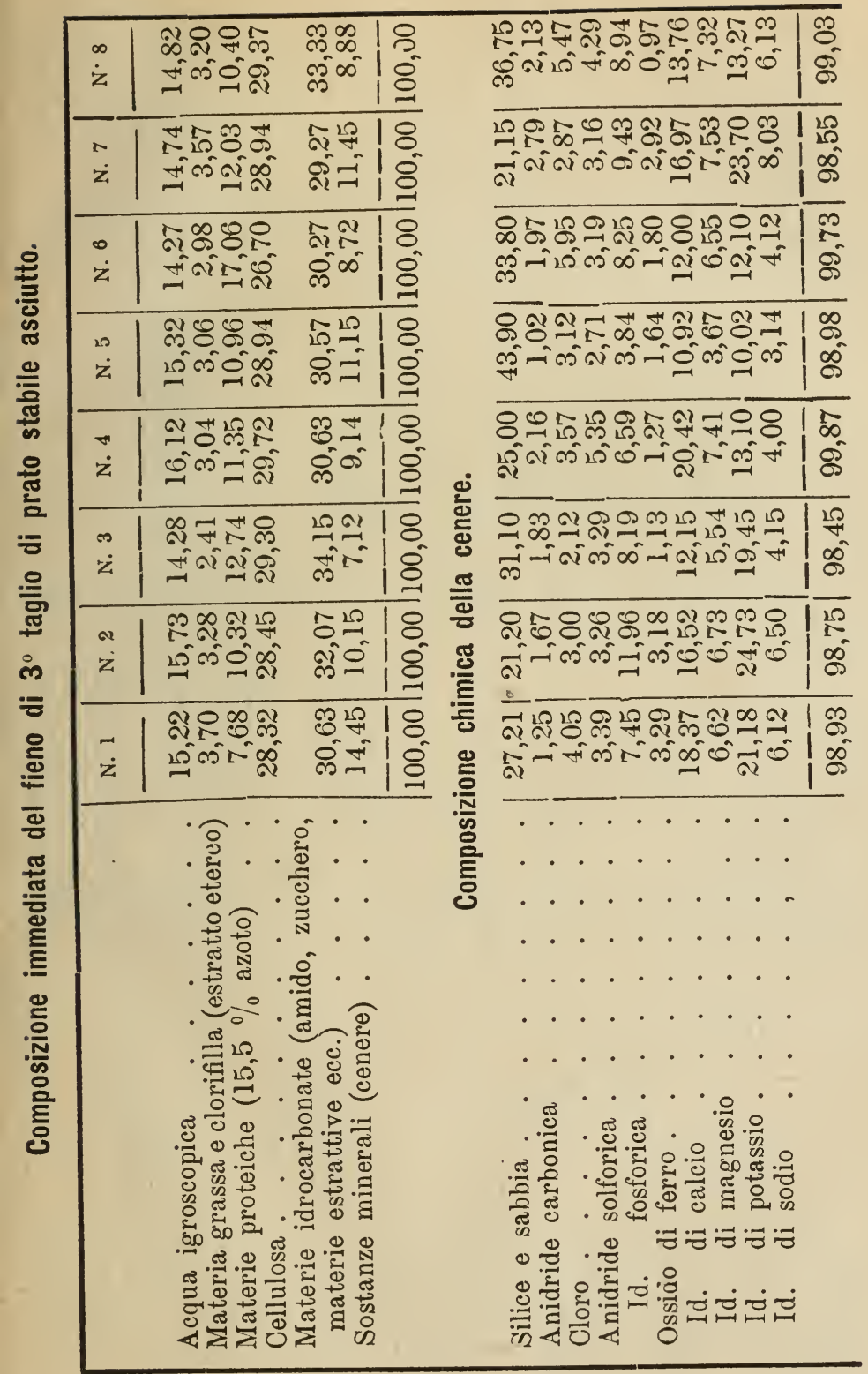


Come venne praticato per l'erba delle marcite, onde potersi fare un giusto criterio sul valore nutritivo di questi foraggi, sarà opportuno di istituire un confronto fra i dati ottenuti con l'analisi chimica dei fieni Novaresi e quelli riportati da altri sperimentatori con fieni di paesi diversi.

Boussingault per esempio, nel suo quadro sulla costituzione delle sostanze vegetali alimentari; attribui al fieno ed al guaime la seguente composizione centesimale.

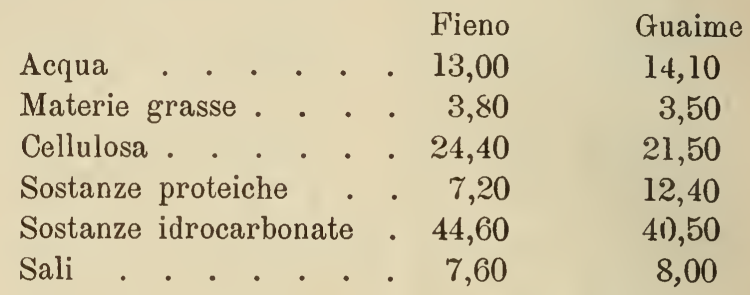

Il Kühn per il fieno maggengo di prato asciutto dà la seguente composizione:

\begin{tabular}{|c|c|c|c|c|c|c|}
\hline Sostanze & proteiche & . & . & $\begin{array}{c}\text { Minima } \\
5,8\end{array}$ & $\begin{array}{c}\text { Massima } \\
18,5\end{array}$ & $\begin{array}{c}\text { Media } \\
8,5\end{array}$ \\
\hline 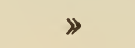 & grasse & . & . & 1,4 & 5,6 & 3,0 \\
\hline$»$ & estrattive $n$ & non & azotate & 22,6 & 50,7 & 38,3 \\
\hline Cellulosa & . & . & . & - 19,7 & 39,9 & 29,3 \\
\hline
\end{tabular}

Il Gohren nel suo bellissimo lavoro sull'alimentazione degli animali domestici, al fieno maggengo e all'agostano assegna la composizione chimica che in appresso:

\begin{tabular}{|c|c|c|c|c|c|}
\hline Acqua & . & & & 14,3 & 15,0 \\
\hline Materie & plastiche. & & & 8,5 & 9,5 \\
\hline$»$ & grasse & & & 3,0 & 3,1 \\
\hline$》$ & idrocarbonat & & & 38,3 & 42,3 \\
\hline Cellulosa & - & $\bullet^{\circ}$ & • & 29,3 & 23,5 \\
\hline Ceneri & . & • & • & 6,02 & 6,6 \\
\hline
\end{tabular}

Finalmente il professore Sestini nel suo interess antissimo studio chimico sui foraggi della campagna Romana, assegna la qui sotto indicata composizione ad otto campioni di fieno maggengo di prato asciutto, raccolti in luoghi e giorni diversi. 


\begin{tabular}{|c|c|c|c|c|c|c|c|}
\hline $\begin{array}{l}\infty \\
\dot{z}\end{array}$ & 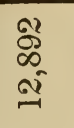 & $\stackrel{\infty}{\sigma}$ & $\begin{array}{l}19 \\
0_{0} \\
\infty \\
\infty\end{array}$ & $\underset{\sigma}{\stackrel{Z}{*}}$ & $\begin{array}{l}\overrightarrow{8} \\
0 \\
00 \\
00\end{array}$ & 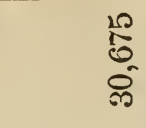 & $\begin{array}{l}8 \\
8 \\
8 \\
8\end{array}$ \\
\hline$\hat{z}$ & 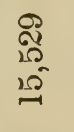 & $\underset{\text { مi }}{\stackrel{0}{二}}$ & 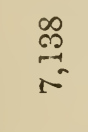 & $\underset{\infty}{\stackrel{\vec{O}}{\infty}}$ & 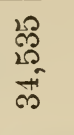 & $\stackrel{\vec{\infty}}{\vec{\Phi}}$ & $\begin{array}{l}8 \\
8 \\
8 \\
8\end{array}$ \\
\hline $\begin{array}{l}\dot{z} \\
\dot{z}\end{array}$ & $\begin{array}{c}\hat{\mathfrak{g}} \\
\stackrel{5}{\sim} \\
\stackrel{5}{\sim}\end{array}$ & $\stackrel{\infty}{\stackrel{0}{0}}$ & $\begin{array}{l}\infty \\
\infty \\
\infty \\
\infty \\
0\end{array}$ & $\frac{\tilde{\sigma}}{\tilde{0}}$ & $\begin{array}{l}\underset{4}{\mathbb{N}} \\
\frac{\pi}{5}\end{array}$ & $\begin{array}{l}0 \\
0 \\
0 \\
0 \\
0\end{array}$ & $\begin{array}{l}8 \\
8 \\
8 \\
8\end{array}$ \\
\hline $\begin{array}{l}20 \\
\ddot{z}\end{array}$ & 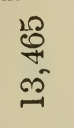 & 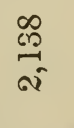 & 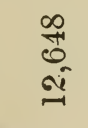 & $\vec{\delta}$ & $\begin{array}{l}\text { 亲 } \\
\vec{b}\end{array}$ & $\begin{array}{l}\overrightarrow{\overrightarrow{0}} \\
\infty \\
\infty\end{array}$ & $\begin{array}{l}8 \\
8 \\
8 \\
8\end{array}$ \\
\hline$\vec{z}$ & $\begin{array}{l}\stackrel{\leftrightarrow}{\sigma} \\
\stackrel{\sim}{\sim}\end{array}$ & 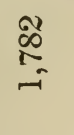 & 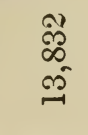 & 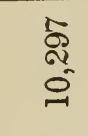 & $\begin{array}{l}\stackrel{0}{0} \\
\text { in } \\
\text { - }\end{array}$ & s. & $\begin{array}{l}8 \\
8 \\
8\end{array}$ \\
\hline $\begin{array}{l}\infty \\
\dot{z}\end{array}$ & $\begin{array}{l}\mathscr{D} \\
\stackrel{2}{9} \\
\stackrel{-}{-}\end{array}$ & $\underset{-i}{\overrightarrow{5}}$ & 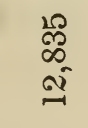 & $\underset{\sigma}{\stackrel{\infty}{\leftrightarrows}}$ & $\begin{array}{l}\stackrel{\mathscr{\sigma}}{\vec{N}} \\
\vec{\infty}\end{array}$ & 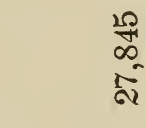 & $\begin{array}{l}8 \\
8 \\
8 \\
8\end{array}$ \\
\hline $\begin{array}{l}\ddot{z} \\
\dot{z}\end{array}$ & 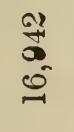 & 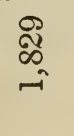 & $\begin{array}{l}8 \\
10 \\
10 \\
9 \\
-1\end{array}$ & $\begin{array}{l}\hat{1} \\
\text { m } \\
\text { - }\end{array}$ & 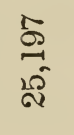 & 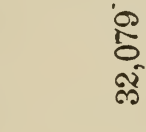 & $\begin{array}{l}8 \\
8 \\
8\end{array}$ \\
\hline $\overrightarrow{\dot{z}}$ & 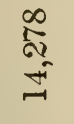 & 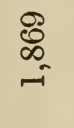 & 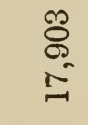 & $\frac{\vec{D}}{\vec{\sigma}}$ & $\begin{array}{l}\overrightarrow{8} \\
8 \\
8\end{array}$ & $\begin{array}{l}18 \\
08 \\
0 \\
0\end{array}$ & $\begin{array}{l}8 \\
8 \\
8\end{array}$ \\
\hline & 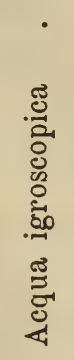 & 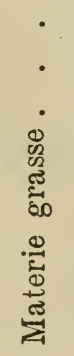 & 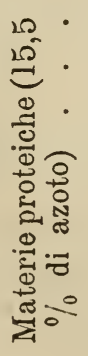 & 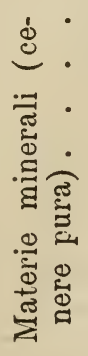 & 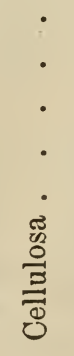 & 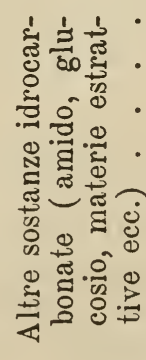 & \\
\hline
\end{tabular}


Istituendo ora il confronto fra tutti questi dati e quelli ottenuti coi fieni Novaresi, chiaramente si vede, in primo luogo, che la proporzione delle materie grasse nei nostri fieni è superiore di quella che si trova nei foraggi della campagna Romana; è per converso presso a poco eguale a quella indicata da Boussingault, e di poco superiore a quella media stabilita dal Kiihn.

Le sostanze proteiche pure nei fieni Novaresi assolutamente abbondano; infatti in tutti i campioni, meno che in quello di terzo taglio indicato col N. ${ }^{\circ}$, si trovano in quantità eguale, ed in alcuni un poco superiore, di quella indicata dal Sestini nei fieni della campagna Romana.

La proporzione invece di principii respiratori (amido, zucchero, sostanze estrattive, ecc. ecc.: in quasi tutti i fieni di prato asciutto analizzati, si trova al disotto della media riportata da Kühn, e di quella trovata da Boussingault e dal Gohren.

La cellulosa, o sostanza legnosa, nei nostri fieni tanto maggenghi che di secondo taglio si trova in giusta proporzione; difatti in cinque soli campioni di maggengo si riscontra in quantità di poco superiore alla rnedia, mentre negli altri vi si rinviene in dose anco inferiore.

La proporzione delle ceneri o principii minerali, pure si vede essere superiore di quella riportata di Boussingault e Gohren.

Il Sestini per converso, ha trovato nei fieni della campagna Romana una quantità di ceneri rappresentata dalle cifre seguenti :

$$
\begin{gathered}
\text { N. } 1-10,72 \\
\gg 2-12,46 \\
》 \quad 3-10,37 \\
\gg \quad 4-11,82
\end{gathered}
$$$$
\begin{array}{rr}
\text { N. } & 5-10,04 \\
》 \quad 6-10,97 \\
\gg 7-9,59 \\
\gg 8-10,61
\end{array}
$$

proporzione che supera di tre la media indicata dal Wolff con 7,78 per $\%$ di fieno secco.

Se nei fieni Novaresi di primo taglio, i principii minerali si trovano in quantità non molto superiore alla media surriferita, è da dire che quelli di secondo e di terzo taglio si riscontrano in dose eccedente. Del resto poi ben si vede come in queste ce- 
neri la potassa non raggiunga la media indicata da Wolff con 25,6 per $\%$ ad eccezione di quelle dei fieni maggenghi segnati con i numeri $4,6,7$, e cosi pure la soda.

L'acido fosforico, che come altrove si è detto, è uno dei più importanti materiali inorganici, nelle ceneri di fieni Novaresi di $1 .^{\circ}, 2 .^{\circ}, 3 .^{\circ}$ taglio, trovasi in proporzione piuttosto abbondante meno che in qualche campione nel quale scarseggia un poco. Abbonda per converso, in queste ceneri, ed in modo straordinario la silice, ma ciò non deve recare grande meraviglia, sapendosi bene dagli agricoltori che i terreni del Novarese sono in generale di natura essenzialmente argilloso-silicea.

Degli altri composti minerali nulla occorre di dire, perché di poca importanza dal lato della nutrizione, ed anco perché gli animali ne ricevono in copia sufficiente dalle acque che ingeriscono.

Ora che abbiamo indicato la composizione dei fieni Novaresi di prato asciutto, che abbiamo veduto come in essi i vari materiali nutritivi si trovano in proporzione sufficiente, meno poche eccezioni, cerchiamo di constatare se possouo soddisfare le esigenze degli animali che se ne cibano, prendendo per guida di questa determinazione, i lavori eseguiti in molte stazioni fisiologiche di Germania da vari rinomati sperimentatori.

Dopo una lunga serie di pazienti esperienze e di analisi chimiche Grouven, Wolff, Gohren, Haubner ed altri giunsero a determinare le quantità di principii plastici, di principii respiratori, di cellulosa e di materiali minerali che occorrono alle varie specie di animali domestici, per mantenersi in perfetta salute e fornire quei prodotti che sono richiesti da ciascuna categoria di bestiame.

Risultato di siffatte esperienze fu, che per le bestie bovine tenute nelle stalle nelle quali la temperatura oscilla tra i $12 .^{\circ}$ e $18 .^{\circ}$ centigradi, occorrono, quando stanno in riposo, e quando non debbono che mantenersi in buono stato senza fornire prodotto alcuno, le seguenti quantità di principii nutritivi per 1000 chilogrammi di peso vivo:

Cellulosa chilogr. 6 - Principii plastici chilogr. 0,9-Principi respiratori chilogr. 7,2. 
Queste sostanze stanno adunque fra di loro nelle proporzioni ponderali che in appresso:

Cellulosa : Principii respiratori $=+$ Principii plastici $=:$ : $3: 4$ e principii plastici $=$ : Principii respiratori $=:: 1: 8$.

Per quanto poi riguarda i composti minerali, è stato ammesso che sono almeno necessari 25 grammi di acido fosforico e 50 grammi di calce; in generale peró non si tiene molto conto di questi materiali nel confezionamento delle razioni, sapendosi bene come nei foraggi sempre si trovino in quantita esuberante.

In base ai dati surriferiti, e che ho tolti dal pregevolissimo lavoro del professore Sestini sui foraggi della campagna Romana, passiano ora a determinare la quantità di principii assimilabili che si trovano nelle razioni formate da solo fieno maggengo o agostano di prato asciutto Novarese.

Le razioni (espresse in valore corrispondente al fieno) del bue in riposo, stabilite dai più autorevoli agronomi sono di chilogrammi 1,66 per $\%$ di peso vivo; perciò in chilogrammi 16,6 di foraggio dei nostri prati asciutti si troveranno i più importanti principi alimentari nella proporzione seguente: 


\begin{tabular}{|c|c|c|}
\hline $\begin{array}{l}= \\
\dot{z}\end{array}$ & 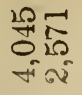 & $\underset{20}{\stackrel{50}{*}}$ \\
\hline$\stackrel{\varrho}{\dot{z}}$ & 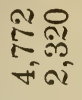 & $\underset{10}{\stackrel{0}{+}}$ \\
\hline $\begin{array}{l}o \\
\dot{z}\end{array}$ & $\begin{array}{l}\hat{9} \\
\overrightarrow{10}\end{array}$ & 赵 \\
\hline $\begin{array}{l}\propto \\
\dot{z}\end{array}$ & 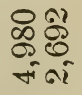 & $\begin{array}{c}0 \\
0 \\
0\end{array}$ \\
\hline$\dot{z}$ & 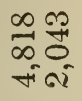 & $\begin{array}{l}\text { مे } \\
20 \\
20\end{array}$ \\
\hline $\begin{array}{l}0 \\
\dot{z}\end{array}$ & 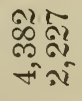 & $\begin{array}{l}5 \\
\infty \\
20 \\
20\end{array}$ \\
\hline $\begin{array}{l}\text { in } \\
\dot{z}\end{array}$ & 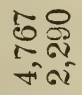 & ְొ \\
\hline $\begin{array}{l}\vec{y} \\
z\end{array}$ & 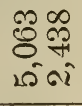 & $\underset{20}{\overrightarrow{+2}}$ \\
\hline $\begin{array}{l}\infty \\
\dot{z}\end{array}$ & 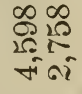 & $\vec{\delta}$ \\
\hline 品 & 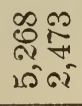 & $\underset{20}{\stackrel{\overrightarrow{2}}{2}}$ \\
\hline$\ddot{z}$ & 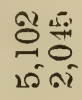 & $\begin{array}{l}\overrightarrow{10} \\
10 \\
20\end{array}$ \\
\hline & \multicolumn{2}{|c|}{ 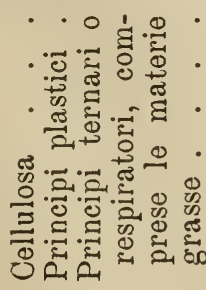 } \\
\hline
\end{tabular}

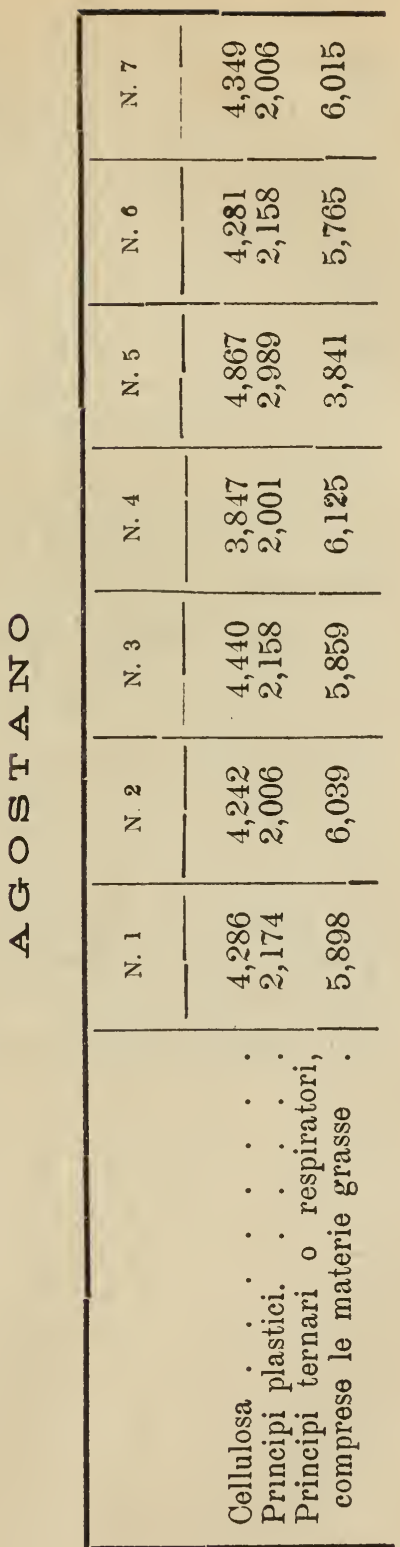


Questi due specchietti ben nettamente dimostrano che nelle razioni di solo fieno Novarese tanto di primo che di secondo taglio, supposto che agli animali suddetti si dia una intera razione, si trovano in copia grande i principi proteici, ma scarseggiano la cellulosa ed i principi respinatori.

E da dire, che a questa deficienza si potrebbe benissimo rimediare aumentando, nella razione fissata, la proporziono della cellulosa e diminuendo quella delle sostanze proteiche. A tal uopo basterebbe sostituire una data quantità di paglia ad una parte di fieno ed aggiungere al miscuglio qualche sostanza ricca di elementi respiratori, quali per esempio, le radici da foraggio.

Siffattamente operando, si giungerebbe ad aumentare nella razione la cellulosa ed i materiali idrocarbonati, a giustamente diminuire i principi plastici e per conseguenza ad avere un mangime provvisto in giusta dose di principi nutritivi, ed anco con molta probabilità di un costo minore.

Per le vacche lattifere e pei buoi da lavoro la comune razione dovrebbe contenere in media chilogrammi 9 di cellulosa, chilogrammi 2,5 di principi plastici e chilogrammi 12,48 di principi respiratori; detta razione per questi animali deve essere di chilogrammi 33,3 per 1000 di peso vivo.

I nostri fieni maggenghi e di secondo taglio, presi nella quantità rappresentata dalla razione stabilita per le vacche lattifere e pei buoi da lavoro, sarebbero provvisti dei principali principi alimentari nella proporzione seguente: 

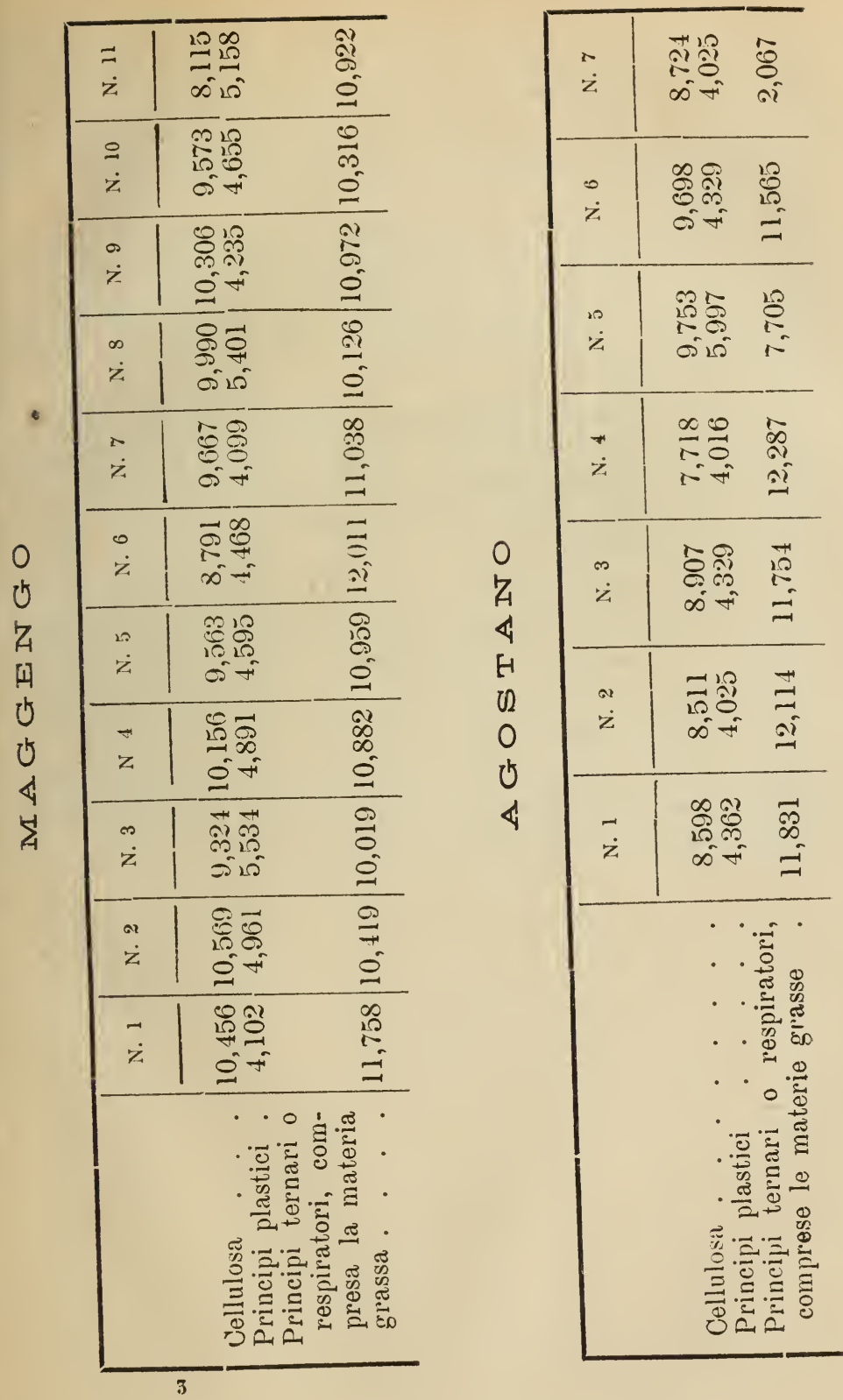
Le vacche lattifere ed i buoi da lavoro con siffatte razion! riceverebbero una quantitá troppo forte di cellulosa, con i fieni maggenghi contrassegnati con i numeri l, 2, 4, 8, 9 e con quelli di N. 5 e 6 di agostano, mentre questo principio si troverebbe in giusta proporzione in tutti gli altri di primo e secondo taglio.

I principi plastici in tutte e due le qualitá di fieni si trovano sempre in abbondanza; scarseggiano invece tanto nei primi che nei secondi i principi respiratori, che nell'organismo delle vacche lattifere hanno una grande influenza sulla produzione e sulla qualità del iatte.

Con fieni silfatti sarebbe adunque buona pratica, specialmente nel caso dell'alimentazione delle vacche lattifere, di aggiungere alla razione una giusta dose di una sostanza ricca di materie grasse e di materiali idrocarbonati, quale per esempio la panella di seme di lino.

La razione occorrente per un cavallo, sempre secondo le in dicazioni fornite da esperimenti rigorosi, dovrebbe contenere chilogrammi 5 di cellulosa, chilogrammi 1,8 di princıpi plastici, chilogrammi 8,7 di principi respiratori, e la quantità di fieno assegnata come razione di un cavallo, si è di 2 chilogrammi per 100 di peso vivo. In 20 chilogrammi di fieno maggengo e di agostano di prato asciutto Novarese, si troveranno adunque i paincipi nutritivi nelle seguenti preporzioni: 


\begin{tabular}{|c|c|c|}
\hline $\begin{array}{l}z \\
\dot{z}\end{array}$ & $\begin{array}{l}\vec{T} \infty \\
\infty \\
\nabla_{0}^{\infty} \infty\end{array}$ & $\begin{array}{l}8 \\
10 \\
0\end{array}$ \\
\hline$\frac{O}{\dot{z}}$ & $\begin{array}{l}10 \% \\
108 \\
100\end{array}$ & $\stackrel{8}{\stackrel{8}{\circ}}$ \\
\hline $\begin{array}{l}0 \\
\dot{z}\end{array}$ & 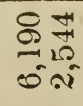 & $\begin{array}{l}8 \\
\text { is } \\
6\end{array}$ \\
\hline $\begin{array}{l}\infty \\
\dot{z}\end{array}$ & 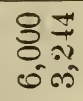 & $\begin{array}{l}\hat{\alpha} \\
\hat{\sigma} \\
0\end{array}$ \\
\hline$\hat{z}$ & $\begin{array}{l}80 \\
80 \\
100 \\
0\end{array}$ & $\begin{array}{l}\text { ర్ } \\
0 \\
0\end{array}$ \\
\hline $\begin{array}{l}0 \\
\dot{z}\end{array}$ & 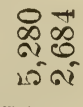 & $=\frac{\vec{N}}{N}$ \\
\hline$\ddot{z}$ & 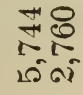 & $\begin{array}{l}0 \\
\infty \\
10 \\
0 \\
0\end{array}$ \\
\hline$\dot{z}$ & $\begin{array}{l}8 \infty \\
8.0 \\
0 \\
0\end{array}$ & $\begin{array}{l}\infty \\
10 \\
i \infty \\
0\end{array}$ \\
\hline$\ddot{z}$ & 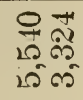 & $\begin{array}{l}\overrightarrow{0} \\
0 \\
0\end{array}$ \\
\hline$\dot{z}$ & 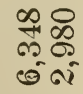 & $\begin{array}{l}\infty \\
10 \\
\vdots \\
0\end{array}$ \\
\hline$\ddot{z}$ & $\begin{array}{l}\infty \\
0 \\
0 \\
0 \\
0 \\
0 \\
0\end{array}$ & $\underbrace{8}_{i=}$ \\
\hline & \multicolumn{2}{|c|}{ 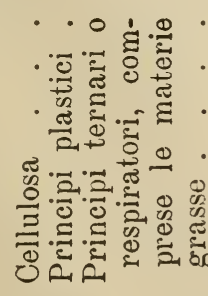 } \\
\hline
\end{tabular}

\begin{tabular}{|c|c|}
\hline$\dot{z}$ & 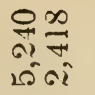 \\
\hline $\begin{array}{l}0 \\
\dot{z}\end{array}$ & 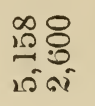 \\
\hline in & $\begin{array}{l}\overrightarrow{0} \hat{ᄋ} \\
\& \\
\dot{0} \sigma i\end{array}$ \\
\hline$\vec{z}$ & 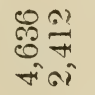 \\
\hline $\begin{array}{l}\infty \\
\dot{z}\end{array}$ & $\begin{array}{l}08 \\
108 \\
100 \\
100\end{array}$ \\
\hline $\begin{array}{l}\alpha \\
\dot{z}\end{array}$ & 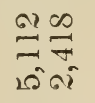 \\
\hline$\vec{z}$ & 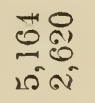 \\
\hline & 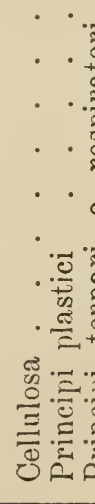 \\
\hline
\end{tabular}


Fatta eccerione pel num. Il di primo taglio e del num. 4 di secondo, in tutte queste razioni abbonderebbero la cellulosa ed i principi plastici, mentre sarebbero un poco scarsi i principi respiratori. Questi fieni da soli non possono adınque dar luogo a delle perfette razioni pei cavalli, e per ottenerle tali eccellente pratica sarebbe quella di sostituire una data quantitá di fieno con una giusta dose di crusca di frumento o di qualche altra sostanza ricca di materie idrocarbonate.

Finalmente é stato stabilito che si debba fornire ai cavalli da tiro destinati a continue fatiche, per 1000 di peso vivo, la razione contenente chilogrammi 7,44 di cellulosa, chilogrammi 3,88 di principi plastici e chilogrammi 12,90 di sostanze idrocarbonate, e che non si debba mai dare loro una quantità di fieno, per 1000 di peso vivo, minore di chilogrammi 36,70.

I fieni Novaresi di primo e secondo: taglio, somministrati ai cavalli da fatica nella proporzione suindicata cederebbero loro i principi nutritivi nelle seguenti quantità: 

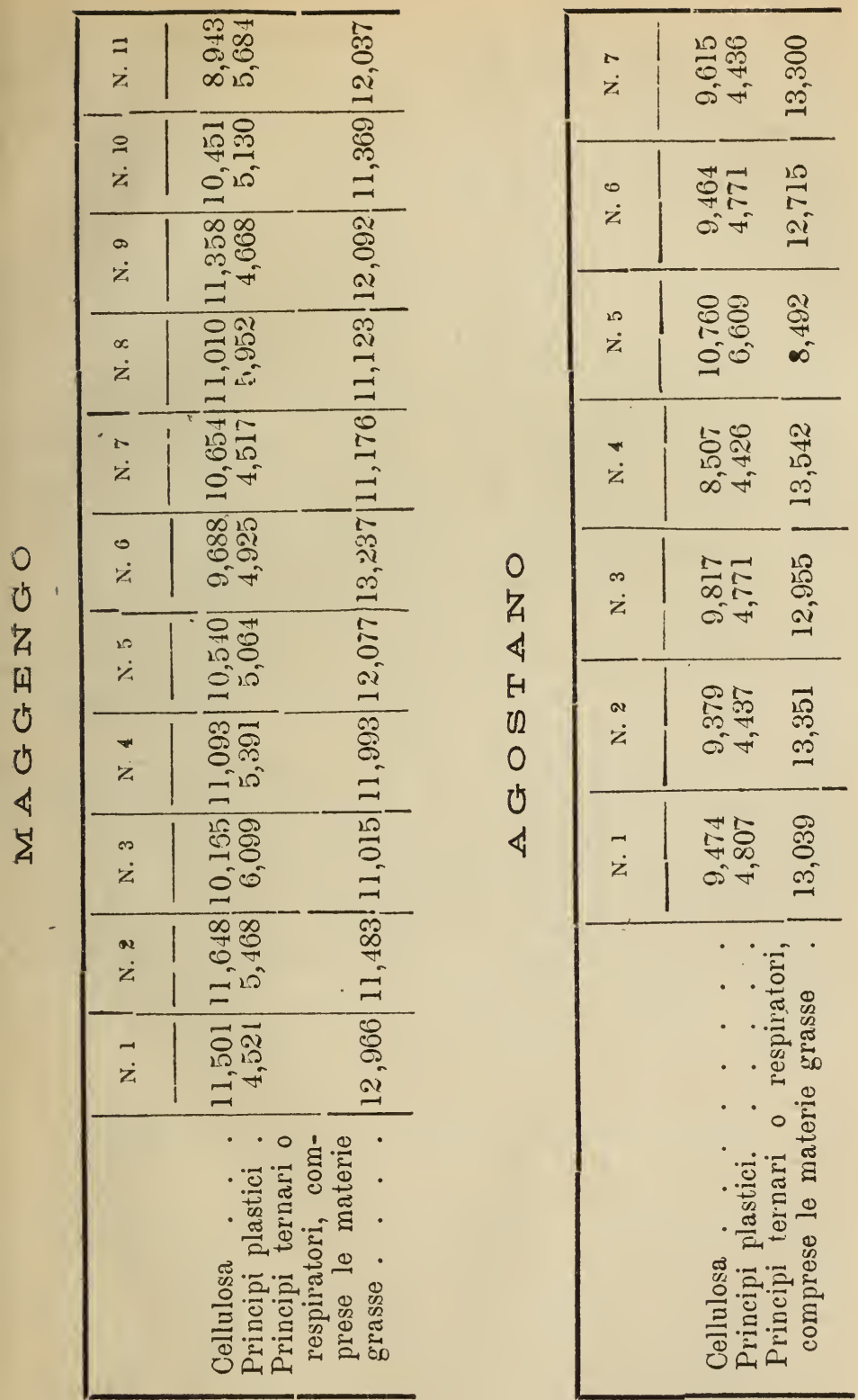
Tali razioni per dire il vero non sarebbero molto adatte pel' fornire un completo nutrimento ad un cavallo da tiro; in esse infatti la cellulosa si trova in quantitá eccessiva specialmente nei fieni maggenghi, le sostanze proteiche, come negli altri casi, abboudano ed i materiali respiratori, meno che nei fien maggenghi segnati con $\mathrm{i} n$. 1 e 6 ed in quelli di n. $1,2,3$, 4, 6, 7 di secondo taglio, sono alquanto scarsi. Perció onde avere con questi fieni una perfetta razione alimentare per un cavallo da tiro, bisognerebbe sostituire una parte di fieno con qualche altra sostanza nutritiva poco voluminosa, ricca in sostanze idrocarbonate e di principi proteici, ma scarsa di cellulosa, quale per esempio l'orzo, le fave, il mais, ecc. ecc. avendo peró sempre la cautela di praticare questa addizione in modo tale, da ottenere una razione giustamente provvista di tutti e tre i materiali necessari per l'alimentazione degli animali.

Le suesposte cifre, sebbene sieno state conseguite con rig'(1rosi e pazientissimi studi, tuttavia non debbono presentare un valore assoluto per gli agricoltori, ma possono essere prese come base e cosi servire di guida nella razionale confezione delle razioni occorrenti al bestiame.

L'agricoltore poi, dopo di averne accuratamente osservati gli effetti, potrà modificarle secondo il caso, ricordando che non tutti gl'individui hanno le medesime facoltà digestive, e che non tutti i materiali alimentari dei foraggi sono in eguale misura assimilabili.

Finalmente si dovrà pure rammentare che per ricavare nell'allevamento del bestiame un buon profitto, non solo è assolutamente necessario di produrre dei buoni foraggi, ma è duopo eziandio che sieno impiegati in modo conveniente.

\section{Fieni di prato irriguo di I. ${ }^{\circ}$ e II. ${ }^{\circ}$ taglio.}

Senza premettere alcuna generalità sui fieni di prato irriguo potendosi riferire a questi quanto venne esposto altrove pei fieni di prato asciutto, credo opportuno di riportare nei qui sotto tracciati specchietti la composizione chimica immediata di n. 9 campioni di fieno maggengo o di primo taglio, e di n. 2 qualità di agostano o di secondo taglio di prati irrigui del circondario di Novara. 


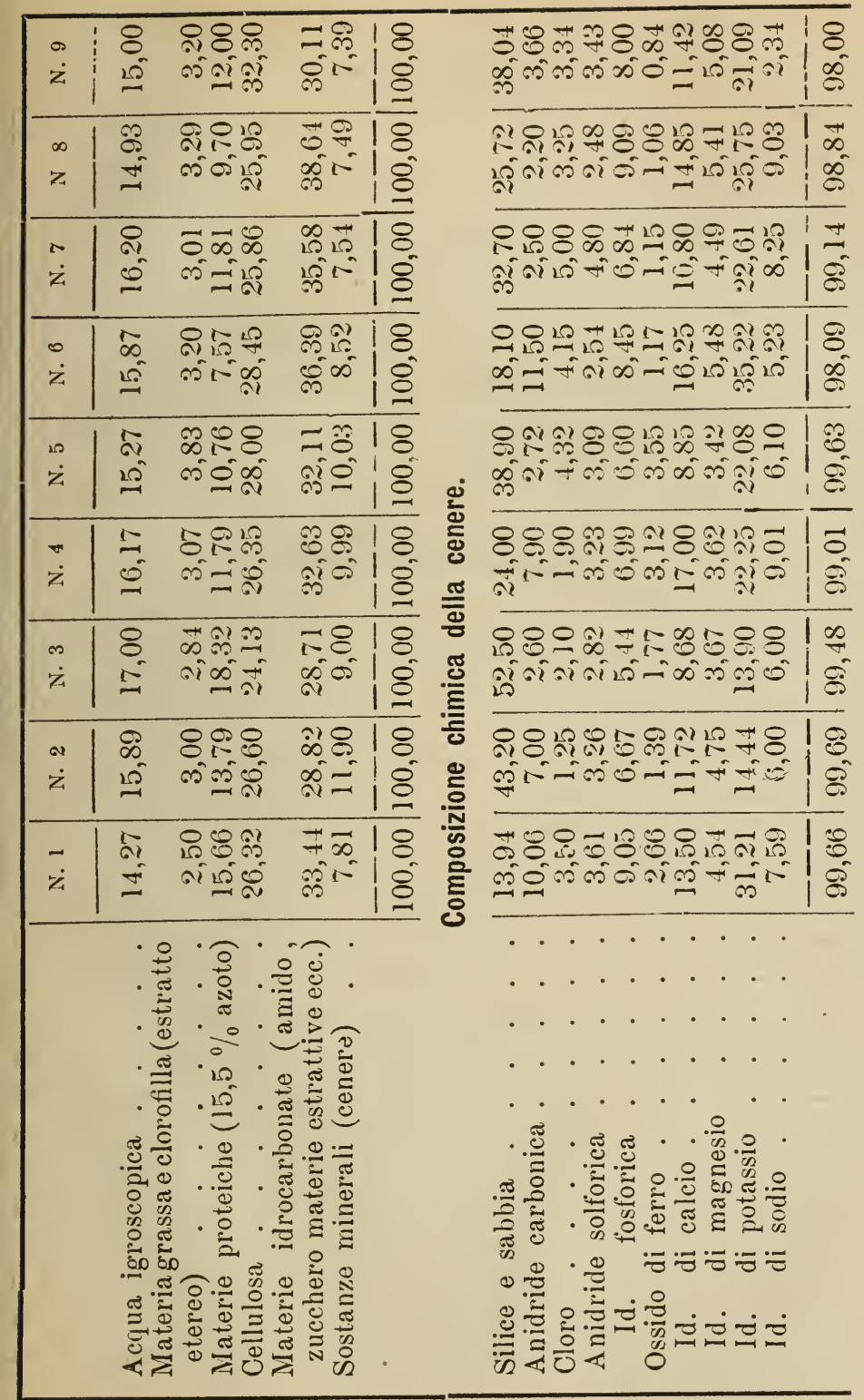




\section{Acqua igroscopica}

Materie grassa con clorofilla (estratto etereo)

Materie proteiche $(15,5 \%$ azoto $)$

Cellulosa

Materie idrocarbonate (amido, zucchero, materie estrattive ecc.)

Sostanze minerali (cenere) ....

\begin{tabular}{|c|c|}
\hline N. 1 & \multicolumn{1}{c|}{ N. 2 } \\
\cline { 1 - 2 } 14,23 & 15,10 \\
3,27 & 3,16 \\
10,82 & 13,20 \\
29,02 & 25,51 \\
35,17 & 34,74 \\
7,49 & 8,29 \\
\hline$-\frac{10}{100,00}$ & 100,00 \\
\hline
\end{tabular}

\section{Composizione della cenere.}

Silice e sabbia.

Anidride carbonica

Cloro .

Anidride solforica.

Id. fosforica .

Ossido ferrico

Id. di calcio

Id. di magnesio

Id. di potassio

Id. di sodio

\begin{tabular}{|r|r|}
33,70 & 26,40 \\
2,31 & 2,19 \\
1,08 & 3,50 \\
3,43 & 2,63 \\
7,95 & 8,97 \\
0,64 & 1,20 \\
10,64 & 16,72 \\
3,71 & 6,31 \\
27,09 & 22,46 \\
08,01 & 8,34 \\
\hdashline 98,56 & 98,72
\end{tabular}

Ponendo ora questi dati a confronto di quelli che vennero riportati per indicare la composizione chimica dei fieni di prato asciutto, ed anco di quelli stabiliti dal Kühn come media, chiaro apparisce che in questi fieni di prato irriguo, le sostanze proteiche, meno qualche eccezione, si trovano in proporzione alquanto minore, sempre peró superiore alla media altrove indicata.

La cellulosa pure se non scarseggia affatto, si trova in dose minore che nei fieni di prato asciutto, mentre le sostanze ternarie (amido, zucchero, materie estrattive, ecc, ecc.) si rinvengono in proporzione alquanto piú.forte, ma sempre peró al di sotto della media. 
Le sostanze grasse sono contenute in questi foraggi in quantità di poco superiore del 3 per $\%$, che è quella fissata dal Kühn come meäia, proporzione eguale a quella rinvenuta nei fieni di prato asciutto.

Se poi si passa allo esame dei principii minerali, tosto si vede che in questi fieni la proporzione totale dei materiali inorganici è di poco superiore alla media, meno che nei campioni segnati c('n i numeri 2, 5; e se si getta lo sguardo sui quadri che rappresentano la chimica composizione delle ceneri, si osserva che nel maggior numero di queste abbonda grandemente la silice, si trovano in giusta proporzione gli alcali (soda e potassa) ad eccezione dei numeri $2-3-9$ di primo taglio, e finalmente che l'acido fosforico, che come altrove abbiamo detto, è materiale inorganico essenzialissimo, supera in quantità, tanto nei campioni di maggengo, meno il numero 3 , quanto nei due di guaime quella stabilitá come media.

Ho creduto opportuno istituire anco pei fieni di prato irriguo questi confronti, allo scopo di far apprezzare questi foraggi, che certamente sono di buona qualità, e che in si gran copia il territorio Novarese produce, e nello stesso tempo per indurre gli agricoltori a ritenere che una inigliore cura nella trasformazione dell'erba in fieno, e la falciatura praticata a tempo giustamente opportuno, darebbero con grande probabilità dei foraggi ancora piú perfetti di quelli che in oggi si hanno.

Del resto negli scritti di Agronomia si trovano indicati vari metodi per trasformare l'erba in fieno dotato del suo profumo speciale e delle migliori qualitá. Non tutti peró i metodi proposti possono essere indifferentemente praticati con soddisfacenti risultanze in qualsiasi paese, concorrendo alla buona riuscita dell'uno o dell'altro, le condizioni di clima, la localitá, le qualità dell'erbe, ecc, ecc. Quanto in proposito senza tema di errare si puó asserire, si è che per avere del buon fieno è duopo che il disseccamento dell'erba falciata avvenga da principio in modo sollecito, quindi che si completi quando il fieno si trova ammucchiato. Se per converso il foraggio rimane troppo a lungo esposto all'azione dell'aria e dell'umidita esso perde il suo particolare e grato profumo, acquista un colore gialliccio, diventa 
meno nutritivo, meno digeribile, e molto meno ricercato dal bestiame.

Finalmente, per quanto riguarda il potere nutritivo dei fieni Novaresi tanto di prato asciutto che di prato irriguo, credo sia mio obbligo di ripetere che per quanto i suddetti foraggi sieno in generale di buona qualità, tuttavia sarà sempre una eccellente pratica quella di razionalmente regolare e completare le razioni di fieno con aggiunta di altri prodotti da foraggio, onde poter fornire agli animali un mangime meglio adatto al loro sviluppo e molto più confacente ai loro bisogni.

\section{Dell'erba medica.}

Questa preziosissima pianta di foraggio appartenente alla famiglia delle Papiglionacee, nornata dai botanici Medicago sativa e generalmente conosciuta nei paesi dell'Alta Italia col nome di Erba spagna, sembra originaria della Media e fu portata in Grecia cinque secoli prima dell'era cristiana da Dario re di Persia. Venne coltivata per la prima volta in Spagna nella metá del $16^{\circ}$ secolo, ma la sua cultura non si è generalizzata che nel $18^{\circ}$ secolo. $\dot{\mathrm{E}}$ questa una pianta foraggiera tanto preziosa e stimata che dallo illustre Cuppari fu classificata come la pianta da foraggio per eccellenza nei climi neridionali di Europa, e dal Lecoteux come « un laboratoire de chimie que le coltivateur peut trasporter sur toute sa ferme et qui peut le dispenser d'acheter les engrais, qui coûteut ordinairement fort cher.

L'erba medica assume un rigoglioso sviluppo nei terreni piuttosto leggieri ma profondi, freschi e fertili, poco importa se argilloso calcarei o argilloso silicei, purchè vi si trovi della calce che ama come tutte le leguminose; cresce inveco stentatamente e fornisce dei poveri tagli nei terreni troppo compatti tanto più poi se la stagione corre arida. Infatti la radice a fittone della medica avendo la proprietá di svilupparsi considerevolmente, ha bisogno di una terra tendente allo sciolto onde potersi interrare ed emettere tutte le sue radichette, che sono 
quelle incaricate di provvedere alla nutrizione di tutta la pianta. Da ció bene si comprende come l'erba medica dovrá essere tanto piú rigogliosa, quanto piú facilmente le radichette potranno svilupparsi e moltiplicarsi, aumentando cosi i mezzi di nutrizione.

Un'influenza notevole nello sviluppo, nella durata,e nella produzione dell'erba medica ha pure la profonditá dello strato arabile. E noto difatti che allorquando il sotto suolo resulta costituito da argilla compatta o da rocce coerentiche le radici di questa pianta non possono attraversare essa cresce stentatamente, da puco foraggio e muore anco, dopo qualche anno.

Del resto tatta eccezione per questo caso pel quale non avvi rimedio alcuno, Ed. Wianne nel suo classico lavoro sulle praterie artificiali scrive, che per rendere un terreno adatto alla coltivazione dell'erba medica si puó ricorrere ad un buon drenaggio, se le terre sono argillose umide e compatte onde risanarle e renderie porose, ad un'abbondante addizione di marna o di calce per correggere quei terreni nei quali manca o scarseggia il calcare, ovvero allo spargimento del gesso nella proporzione di 1200 chilogrammi per ettare nei terreni argillososilicei. Vi sono molti agricoltori, scrive sempre il Wianne, nei quali è radicata la credenza che essendo l'erba medica una pianta bonificatrice del suolo, non debba aver bisogno di un terreno molto pingue, ed in appoggio di questa loro idea citano qualı:he esperimento bene riuscito in terreni di mediocre qualità. ¿̇ da dire peró che questo è un madornale errore, contro il quale è duopo che gli agricoltori si mettano bene in guardia per non rimanere delusi e danneggiati.

$\dot{\mathrm{E}}$ vero che la medica é una pianta che arrichisce il suolo, ma ció avviene soltanto quando trova in abbondanza nel sotto suolo i materiali propri per renderlo piú fertile, perchè in questo caso le sue radici li attingono nelle parti più profonde del terreno per portarli alla superficie.

Non debbosi ancora dimenticare che l'erba medica, come tutti gli altri vegetali, per svilupparsi e dar foraggio, non solo ha bisogno dei cosidetti elementi organici (carbonio, idrogeno, azoto ossigeno), ma richiede eziandio una huona dose di materiali inorganici, e se i primi vengono da esse attinti per la 
massima parte dall'atmosfera, i secondi il solo terreno è quello clie glieli fornisce, rendendosi cosi sempre più povero di principi minerali necessari alla nutrizione della pianta. Chə ció poi sia vero lo dimostra il fatto che questo foraggio, detto bonificatore del terreno, non pui prosperare sullo stesso suolo che dopo un lungo riposo del terreno stesso, a meno che non si voglia isterilirlo tanto da renderlo per lunghi anni incapace di produrre questa leguminosa.

L'esperienza ha ancora fatto conoscere che un campo non puó essere trasformato in novello medicajo, che dopo un periodo di riposo lungo quanto quello nel quale ha prodotto dell erba medica. Resta cosi chiaramente dimostrato che quanto viene considerato come un miglioramento, non è che la preparazione degli elementi nutritivi che si trovano nel sotto suolo allo stato latente, per le piante che succedono. L'erba medica infatti attinge con le sue potenti radici dal sotto suolo dei materiali che vi rimarrebbero inerti e improduttivi, li porta nello strato arabile dove servono per la nutrizione di altre piante; il trifoglio agisce nello stesso modo, ma con minore energia.

Sotto questo punto di vista si ha infatti bonificamento, ma questo non è che fittizio, inquantochè gli elementi minerali che entrano nella costituzione delle piante provengono dal terreno, il quale rimane tanto piú impoverito quanto più numerose sono le raccolte.

Si puó adunque concludere col dire che se l'erba medica, come molte altre leguminose che vengono coltivate per foraggic, bonifica lo strato arabile per lo azoto che attinge dall'aria e che abbandona quindi al suolo coi suoi detriti, e per le materie minerali che dal sotto suolo porta alla superficie, essa in generale lo spossa dei suoi piú importanti principi inorganici. Perció se non si vuole diminuire la ricchezza del terreno, è assolutamente necessario restituirgli questi materiali col mezzo di adattati concimi.

La durata di un medicajo generalmente da noi non è superiore di 5 o 6 anni, ma potrebbe vivere anco di piú, se a maggiore profondità venisse lavorato il terreno.

Per avere un buon campo ad erba medica si letama e si ara profondamente il terreno prima dei geli; in marzo poi, 
quando non soffia il vento, si sparge alla volata la semente crivellata e per quanto piú possibile separata dalla cuscuta, nella proporzione di chilogrammi 25 a 30 per ettaro, unita ad un cereale, per esempio l'avena, ma separatamente l'uno dall'altra.

Nel primo anno si ottiene un taglio abbondante nei primi giorni di agosto ed un discreto alia fine di autunno; negli anni successivi si falcia quattro o cinque volte ad intervalli di 40 o 45 giorni. Si pratica il primo taglio negli ultimi giorni di aprile, il secondo ai primi di giugno, il terzo nel mese di luglio, alla fine di agosto il quarto, e se il terreno è molto fertile nell'ottobre si eseguisce un ultimo taglio.

La trasformazione dell'erba medica in fieno esige molte cautele onde evitare la caduta delle foglie nelle quali, come lo dimostrano i quadri d'analisi che qui in appresso si riportano, sono contenuti in abbondanza i principi nutritivi. Non bisogna adunque rimestare tanto il foraggio quando è secco, ma bensi conservarlo nel fienile legato in fasci.

Ja pioggia altera profondamente il fieno di erba medica inquantochè dilavandolo gli fa perdere una grande quantitá di princifi nutritivi, diventa legnoso e non piú ricercato dal bestiame. Nelle annate di abbondanza di fieno di prato naturale si preferisce generalmente di fornire gli animali l'erba medica allo stato verde, in particolar modo quella dei tagli primaverili, ponendo in pratica ben inteso tutte quelle cautele che sono indicate per impedire il meteorismo nel bestiame. I tagli estivi invece vengono trasformati in fieno, che sebbene di qualita inferiore a quello delle buone gramiacee, tuttavia è molto gradito e nutritivo.

I vari saggi di erba medica che vennero sottoposti all'analisi chimica furono tolti da floridissimi medicaj di due, tre e quattro anni in località diverse, quelli di primo taglio nei giorni 23, 26 e 28 di aprile dell'anno 1880 e quelli di secondo taglio nei giorni 10, 15 e 17 di giugno dello stesse anno.

Appena portati in laboratorio questi diversi campioni di foraggio procedetti tosto alla determinazione dell' acqua in essi contenuta, e ciós ponendone una data quantità alla stufa riscaldata $a+100$ fino a peso costante. Il rimanente veniva poi 
disseccato all'ombra, e nel fieno di medica siffattamente ottenuto praticai ancora la valutazione dell' acqua igroscopica, quindi vennero eseguite le altre operazioni onde avere l'analisi completa della pianta.

Altri campioni di questo foraggio, presi sempre nel territorio Novarese in localitá diverse, furono trattati diversamente: cominciai col separare accuratamente le foglie dagli steli, quindi dopo di avere con rigore determinata l'acqua in ambedue queste parti, tanto allo stato verde che allo stato di fieno, ne feci l'analisi completa.

Ho creduto eccellente pratica questa divisione, per dimostrare con l'esperienza alla mano agli agricoltori che la massima quantitd di principi nutritivi risiede nelle foglie di questa pianta foraggiera, e che per conseguenza se nella sua trasformazione in fieno non si ha la cura d'impedire il disperdimento delle foglie, in luogo di fornire agli animali un foraggio ricco di principi plastici e respiratori, si dá loro un mangime abbonlante di cellulosa e poco digeribile.

I quadri d'analisi che riporto indicano, i primi la composizione chimica immediata dell'erba medica presa in complesso tanto allo stato verde che allo stato di fieno, i secondi la composizione delle foglie e degli steli separatamente di questa stessa pianta appena falciata, e dopo il disseccamento all'ombra. Prima peró di tracciare questi quadri, onde si possa fare un confronto tra $\mathrm{i}$ dati da me ottenuti e quelli indicati da altri sperimentatori, credo utile di trascrivere in un quadro i resultati analitici che i signori ingegnere Tito Pasqui agronomo e dettore Alessandro Pasqualini Direttore della Stazione Agraria di Forli, ottennero con l'erba medica del Bolognese, e quellı riportati dal Gohren nel suo lavoro, piú volte citato, sull'alimentazione del bestiame.

Pasqui e Pasqualini Pianta verde

Lequa . . . . . 77,840

Sostanze proteiche . 6,187

Materie grasse . . 0,359

Materie idrocarbonate 10,099

Cellulosa . . . 3,595

Ceneri . $: \quad . \quad 1,924$

\section{GoHren}

Pianta verde Pianta secca

75,3
4,5
0,7
8,4
9,3
1,46

16,4

14,4

2,8

25,7

34,7

7,46 
I primi due scienziati dimostrarono anco sperimentalmente che in queste piante $\mathrm{i}$ materiali nutritivi si trovano in maggior copia quando la fioritura è completa, ed appena iniziata la formazione dei frutti, ed in appoggio di questa loro asserzione portano il seguente specchietto che comprende l'analisı di tre campioni di erba medica raccolti nella stessa localitá ma in epoche diverse, e perció in diverso grado di maturazione.

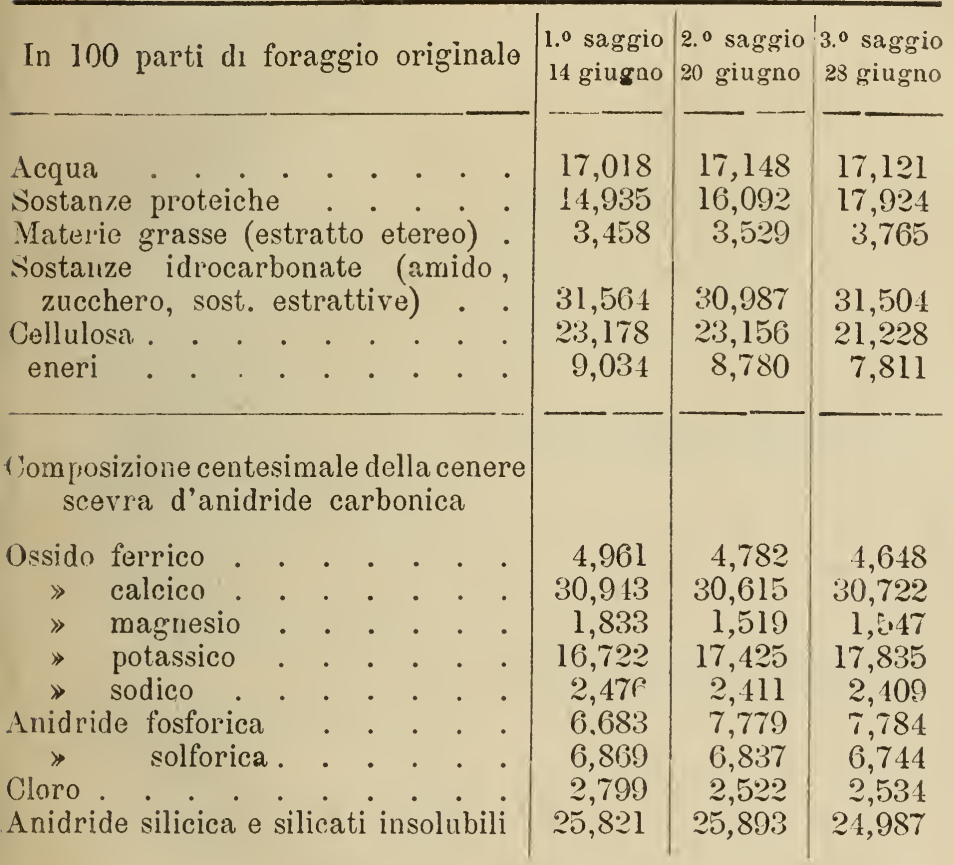

Ecco ora qui in appresso i quadri che secondo le mie analisi scrupolosamente eseguite, stanno a rappresentare la composizione immediata della medica coltivata nel Novarese e quella delle ceneri o sali minerali in essa contenuti. 


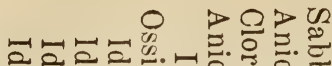

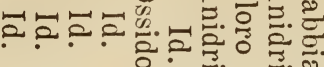

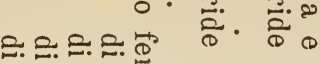

ข็

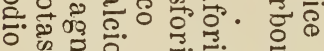

क

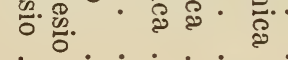

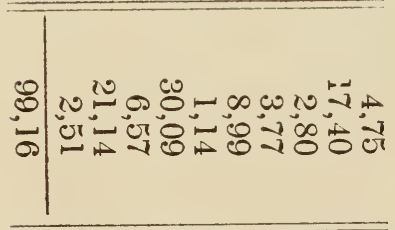

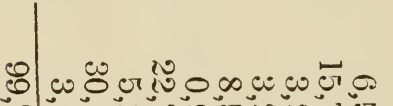
雨

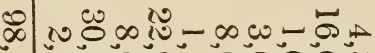

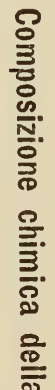

8

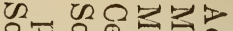

등.

के है है के है

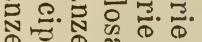

ब

В

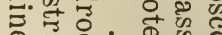

क्ष

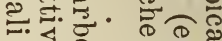

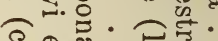

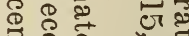

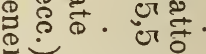

(⿻)

ก

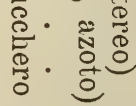

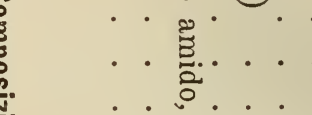

$8 \mid$ ก๊

का w - क ए क -

- 0 iv

81 जा ज记

ह1:

1)

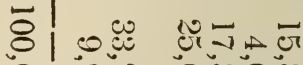

ठ유.

8!

81 जु

हा

8180 जाषच

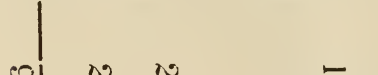

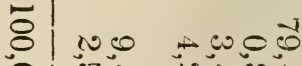

ᄋ10

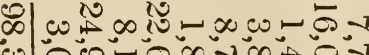

क \&

है: 


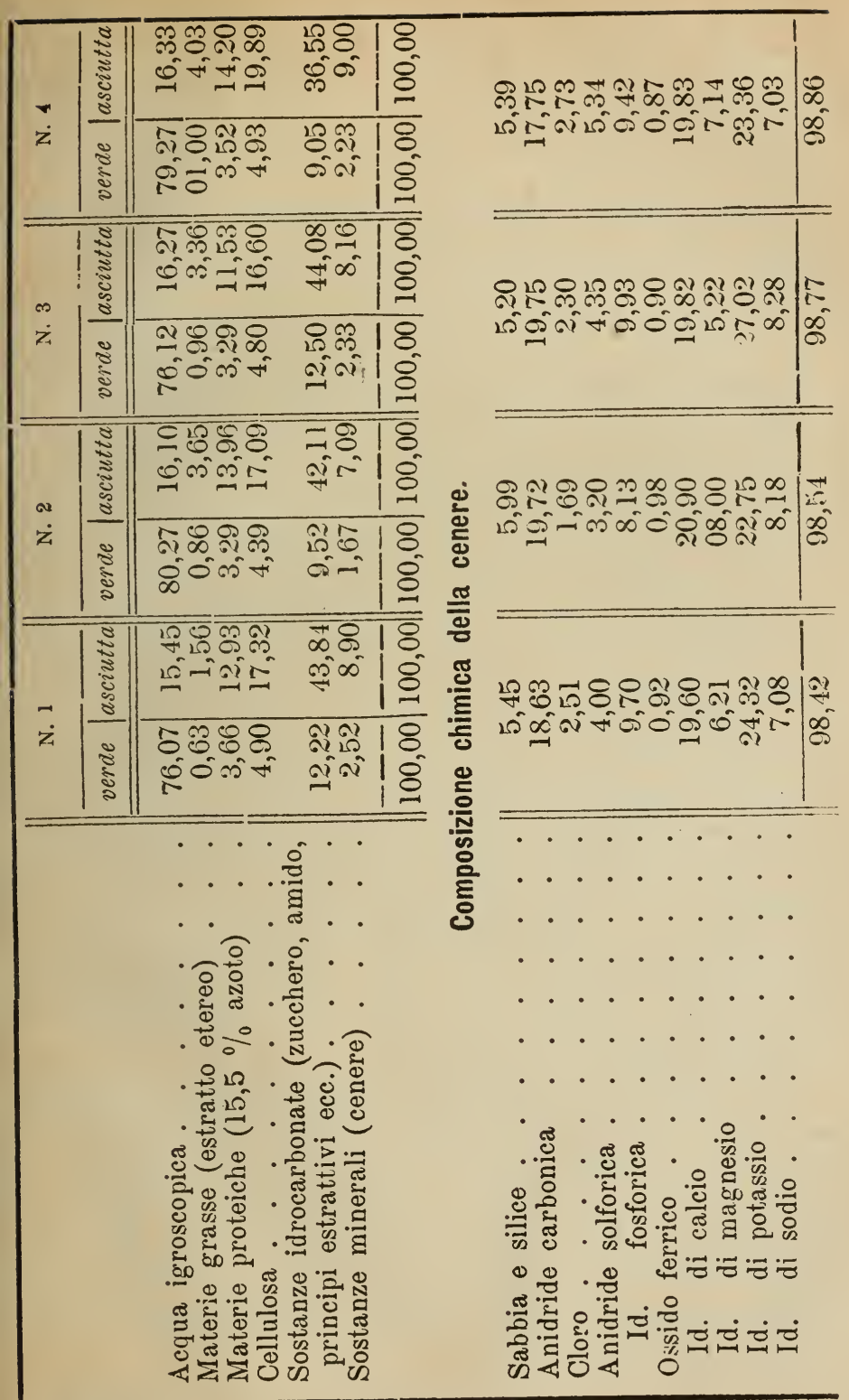


Nelle nostre mediche di $1 .^{\circ}$ taglio, come si puó vedere cor frontando i vari quadri analitici, le materie protiche si trc vano in proporzione superiore di quella indicata per le medich Bolognesi ed anco per le altre, ed è pari a quella stabilità do Kühn per l'eccellente fieno di prato; in quelle di $2 .^{\circ}$ taglio in vece scarseggiano alquanto, ma tale diffetto probabilmente di pende dalla non completa maturità del foraggio analizzato ch diffatti venne falciato prima della sua totale fioritura.

Le sostanze grasse si trovano in quasi tutti i saggi in giu sta proporzione, meno che nel campione di $2 .^{\circ}$ taglio segnat col numero 2, nel quale non raggiungono il 2 per $\%$. Le altr sostanze ternarie o respiratorie si trovano più in abbondanz: nelle mediche di secondo taglio che in quelle del primo, nelle quali peró raggiungono la giusta dose. In quanto poi alla cel lulosa, bene si osservava come nelle mediche di primo taglio $s$ trovi in maggiore quantità che in quelle di secondo, ma an che riguardo a questo principio è da dire, che questo diffett deve essere attribuito alla non matura nza completa del forag gio di $2 .^{\circ}$ taglio.

I principii minerali piú importanti, quali la potassa, la calce e l'acido fosforico, si trovano nelle ceneri dell'erba medica No varese in yuantità piuttosto forte; infatti la potassa sensibil mente in proporzione di quella rinvenuta nelle mediche Bolognesi cosi pure dicasi dell'anidride fosforica. La calce, per converso, specialmente nei secondi tagli, diffetta un poco, ma ciỏ che piú sorprende si $\dot{e}$ il fatto dell'abbondanza della silice nelle ceneri del foraggio Bolognese, nelle quali si trova nella proporzione del 26 per $\%$, mentre nelle nostre non si riscontra che in una quantità inferiore di 8 per cento.

Dai risultati delle praticate ricerche, mi credo autorizzato di poter asserire che l'erba medica coltivata nel Novarese costituisce un eccellente foraggio ricco in principii nutritivi, e che se da tutti gli agricoltori si avesse la cautela di falciare questa pianta quando completamente ha sbocciatı i suoi fiori ed appena iniziata la formazione del frutto, il foraggio suddetto sarebbe ancora di migliore qualità e giustamente provvisto di tutti i materiali alimentari. 
Veggasi ora la composizione chimica delle foglie e degli steli, separatamente, di altri campioni di erba medica di $10^{\circ}, 2 .^{\circ} e$ 3. ${ }^{\circ}$ taglio. 
Composizione immediata del

Erba modica verde.

Acqua

Materie grasse (estratto etereo)

N. 1

Sostanze proteiche (15,5\% azoto) .

Cellulosa

Sostanze idrocarbonate (zucchero, amido, ecc.)

Sostanze minerali (cenere)

\section{Erba medica asciutta.}

Acqua igroscopica

Sostanze grasse (estratto etereo)

Materiali proteici $(15,5 \%$ azoto . . . . Cellulosa

Sostanze idrocarbonate (zucchero, amido, ecc.) Sostanze minerali (cenere)

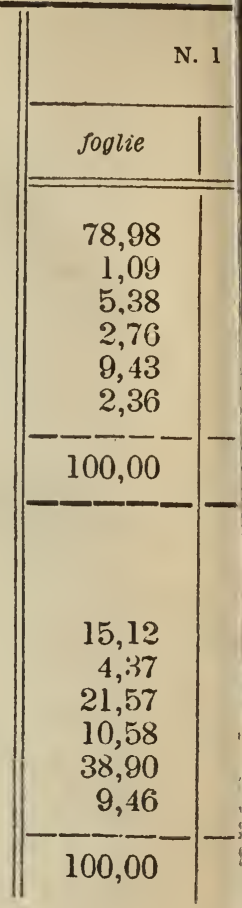

Com

Sabbia e silice

Anidride carbonica Cloro

Anidride solforica

Id.

Id. di calcio

Id. di magnesio

Id. di potassio

Id.

di sodio

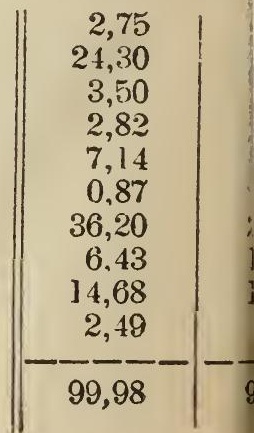


Composizione immediata delle

Erba medica verdo.

Acqua

Materie grasse (estratto etereo)
Sostanze proteiche $(15,5 \%$ azoto)
Cellulo Cellulosa

Materie idrocarbonate (zucchero, amido, ecc.) Sostanze minerali (cenere)

\section{Erba medica asciutta.}

Acqua igroscopica

Materie grasse (estratto etereo)

Sostanze proteiche $(15,5 \%$ azoto $)$. . . Cellulosa

Materie idrocarbonate (zucchero, amido, ecc.) Sostanze minerali (cenere)

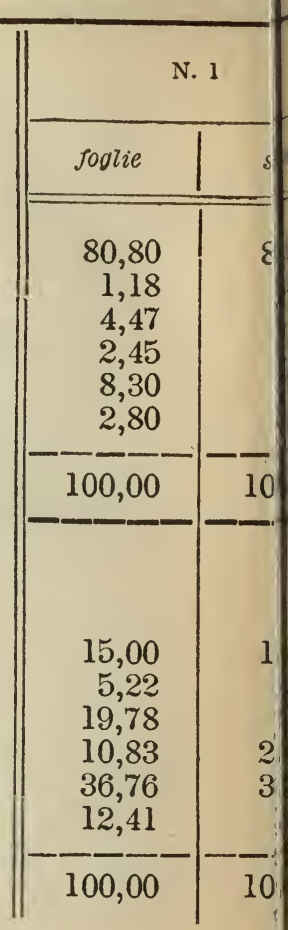

Compo

Sabbia e silice

Anidride carbonica Cloro

Anidride solforica

Id. fosforica

Ossido di ferro

Id.

di calce

Id.

Id. di potassio

Id.

di sodio

\begin{tabular}{r|r}
3,00 & 1 \\
21,75 & 22 \\
0,62 & 1 \\
6,26 & 3 \\
8,44 & 7 \\
0,74 & 1 \\
18,28 & 15 \\
5,56 & 3 \\
28,64 & 36 \\
5,54 & 7 \\
\hline 98,83 & -7
\end{tabular}


Composizione immediata delle

\section{Erba medica verdo.}

\section{Acqua}

Materie grasse (estratto etereo)

Sostanze proteiche $(15,5 \%$ azoto)

Cellulosa

Materie idrocarbonate (zucchero, amido, ecc.)

Sostanze minerali (cenere)

\section{Erba medica asciutta.}

Acqua igroscopica

Materie grasse (estratto etereo)

Sostanze proteiche $(15,5 \%$ azoto $)$. : :

Cellulosa

Materie idrocarbonate (zucchero, amido, ecc.)

Sostanze minerali (cenere.

N. 1

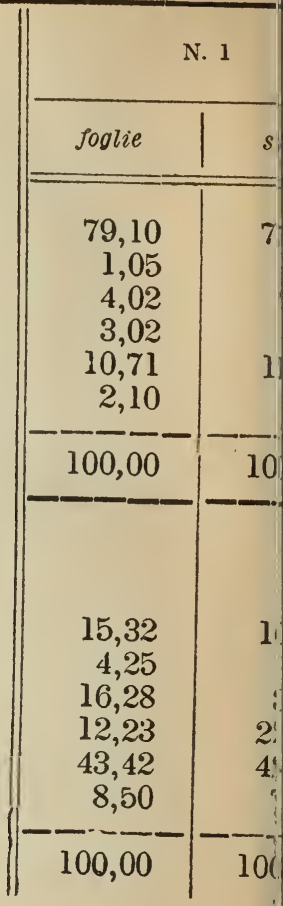

Compo

Sabbia e silice

Anidride carbonica Cloro

Anidride solforica.

Id. fosforica

Ossido di ferro

Id. di calcio

Id. di magnesio

Id. di potassio

Id. di sodio.

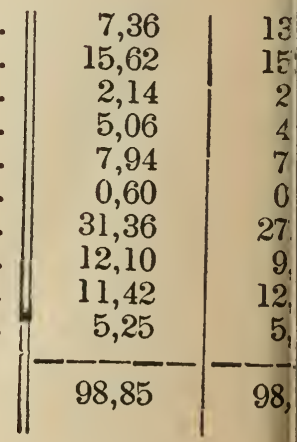




\section{Del Trifoglio.}

Il trifoglio rosso nomato dai botanici Trifolium pratense $\dot{\mathrm{e}}$ una delle piú importanti piante da foraggio della famiglia delle leguminose che estesemente viene coltivata anco nei nostri paesi.

La sua facile coltura, la gran copia di foraggio che fornisce, la sua eccellente qualità, e la sua breve vita lo fanno entrare nelle rotazioni a corto periodo; costituisce infatti il trifoglio la base dell'avvicendamento quadriennale composto generalmente di culture sarchiate, di grano, di trifoglio e di canepa.

Il trifoglio cresce rigoglioso nei climi temperati e nebbiosi, ma sopporta anche i freddi intensi, purchè seminato in terreni freschi e non umidi. La sua radice fusiforme e munita di un gran numero di fibrille esige un terreno che gli permetta di svilupparsi a sensibile profonditá, perció il suolo su quale si semina il trifoglio deve essere non troppo compatto e profondo; non troppo compatto perchè le piccole radichette possino stendersi, profondo perchè il fittone o radice priucipale possa interrarsi ed attingere il suo nutrimento dagli strati più bassi.

In quanto poi alla natura chimica dei terreni $\dot{e}$ da ricordare che il trifoglio ama quelli di natura calcarea non sprovvisti di alcali; poco importa del resto se la calce nelle terre a trifoglio si trovi naturalmente o vi sia introdotti sotto forma di marna, di gesso o di calce spenta. I terreni schistoso-granitici, e quelli da frumento, purchè abbastanza profondi sono i più adatti per lo sviluppo di questa pianta foraggiera.

Il trifoglio sebbene collocato i:el numero delle piante miglioranti, tuttavia ha bisogno di ingrassi, perchè se è vero che esso attinge la massima parte del suo azoto dall'aria atmosferica, che abbandona al terreno, dopo compiuto il suo ciclo vegetativo, le radici che contengono secondo le analisi di Corbeiller 1/100 del proprio peso di azoto, ed una grande quantità di fiorume e di detriti, è pur vero che non prende i suoi principii minerali che dal terreno stesso. Il trifoglio adunque nonostante che rubi molto all'atmosfera, non impoverisce le terre meno delle altre piante, e ciò spiega perchè sotto questa coltivazione si af- 
fatichi di molto il suolo e sia necessario lasciar passare degli anni prima di riseminarlo sullo stesso campo.

Allorquando si coltiva questa pianta sopra un terreno spossato nel quale il foraggio stenta ad accrescersi, è buona pratica quella di operare una discreta concimazione in copertura nell'autunno o nell'inverno, senza peró eccedere di troppo nello ingrasso, perché in tal caso si solleciterebbe lo sviluppo precoce della pianta, che poscia potrebbe venire danneggiata dalle brine.

Sono noti da molto tempo agli agricoltori i sorprendenti effetti del gesso sulle leguminose in generale ma in particolar modo poi sul trifoglio; nondimeno non sono rari i casi di completo insuccesso, e se l'efficacia del gesso non puó essere posta in dubbio, non si conoscono peró ancora bene le cause che concorrono molte volte a renderla nulla.

In generale il gesso produce soddisfacenti effetti nei terreni secchi e fertili, e secondo lo iliustre chimico Emilio Wolff questo composto di solfo, ossigeno e calcio agisce soltanto quando la pianta prospera bene anco senza il suo aiuto, perchè non fornisce ad essa alimento alcuno, ma dispone i materiali del terreno ad agire piú sollecitamente. Il gesso adunque, secondo lo sperimentatore surramentato, non fa l'ufficio che di solvente delle materie nutritive che sono contenute nelle terre, quali lo azoto, la potassa, l'acido fosforico, ecc, ecc.

Wolff paragonando il trifoglio gessato e quello ottenuto senza gesso, trovò che il primo aveva maggiore volume ed era piú ricco d'acqua del secondo. Ridotto in fieno peró non riscontró piú alcuna differenza di volume fra le due qualitá di trifoglio, ma quello gessato conteneva maggior copia di sostanze proteiche, fatto che patentemente sta a dimostrare che il trifoglio governato con gesso aveva assorbito, durante il periodo di sua vegetazione, una maggiore quantità di materiali azotati.

Del resto il gesso non deve essere sparso sul campo che allorquando le piante coprono il suolo e le loro foglie sono umide per leggera pioggia od anco per rugiada. La primavera é l'epoca piú opportuna per l'ingessatura, quando peró non sia da temersi la brina.

Per avere poi un buon raccolto qualunque sia il clima o il terreno sul quale si vuole seminare il trifoglio, $\dot{e}$ assolutamente 
duopo che la terra sia ben preparata e pulita onde lo sviluppo della pianta foraggiera non venga disturbato dalle cattivo erbe. In un campo non bene lavorato ed erpicato, il trifoglio cresce stentatamente e puó anche scomparire nel momento che il seme germoglia.

Il trifoglio quasi sempre si semina nella prima decade di marzo, nella quantità di 18 o 20 chil. per ettaro in mezzo al frumento che venne seminato nel precedente ottobre; ritardando di troppo la semina si correrebbe il rischio di non poter più praticare la rastellatura o erpicatura che deve coprire il seme, troppo avanzato essendo lo sviluppo del grarı.

Nel novello trifogliaio si puó fare un primo taglio tra la fine di ottobre ed il novembre, ed avere cosi un mangime verde in un momento nel quale vi ha penuria di foraggi. Nel secondo anno, se vi è scarsitá di mangimi freschi, praticasi un primo taglio nell'aprile sebbene la pianta non è in fiore, altrimenti in maggio.

Alla fine di giugno si farà il secondo taglio e nell'agosto falciasi l'ultimo, che per solito viene fornito fresco al bestiame, quindi senza aspettare, come erroneamente fanno molti agricoltori il settembre ed anco l'ottobre, si rompre il prato onde far perire le erbe dannose, e si prepara la terra per la cultura del grano.

La trasformazione del trifoglio verde in fieno, esige come l'erba medica, delle grandi cautele, onde le foglie nelle quali, come patentemente lo dimostrano le mie analisi risiede la massima quantità dei principii nutritivi, non si stacchino e vadino perdute.

Falciato adnnque che sia il trifoglio ed appassito, non si deve smuovere spesso come si fa dell'erba dei prati, ma buona pratica si è quella di affastellarlo in grosse masse e abbandonarlo per due giorni a sé stesso onde la fermentazione cominci e per questa ll foraggio acquisti l'odore del miele. Si apre poi la massa e si lascia asciugare alquanto, quindi si affastella di nuovo avendo cura di porre allo estreno la parte che si trovava nello interno, e si abbandona ancora alla fermentazione.

Quando questa é abbastanza sviluppata si stende il foraggio e si dissecca con prontezza. Il fieno di trifoglio preparato con 
questo metodo ha un colore bruno, non ha un bell'aspetto ma il bestiame se ne ciba con aviditá preferendolo ad altro foraggio. Secondo Boussingault, il rapporto fra il furaggio secco ed il prodotto verde varia a seconda del grado di vegetazione della pianta al momento della falciatura; infatti chil. 100 di trifoglio falciato prima della fioritura, danno chil. 21,200 di fieno secco e chil. 35 o 36 di foraggio secco.

Quando il trifoglio è verde, è molto aggradito dal bestiame e favorisce l'ingrassamento e la produzioue del latte, peró se alle vacche lattifere si somministra del solo trifoglio verde, il latte assume un sapore disaggradevole. I cavalli pure lo mangiano avidamente quando è verde, ma nutriti con questo solo foraggio s'indeboliscono di forze, senza contare poi che tanto nel caso dello bestie bovine che dei cavalli si possono correre dei gravi pericoli coll'alimentazione a solo trifoglio, il quale come è noto, produce il meteorismo, specialmente se il bestiame pascola alla mattina prima che sia evaporata la rugiada.

Riguardo poi alla composizione chimica di questa pianta foraggiera Boussingault vi assegna la seguente:

Pianta fresca

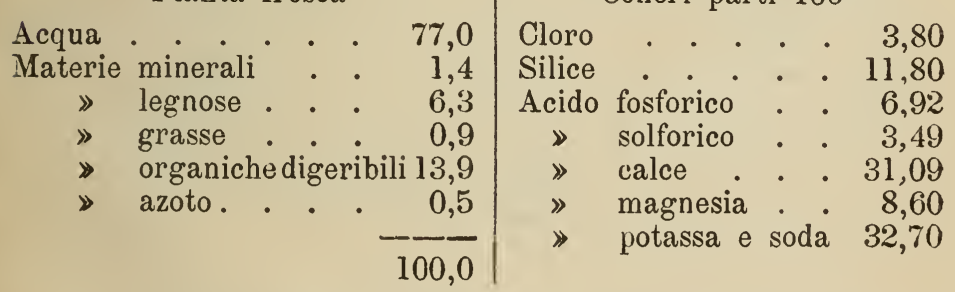

Il Gohren pel fieno di trifoglio dá la composizione che in appresso :

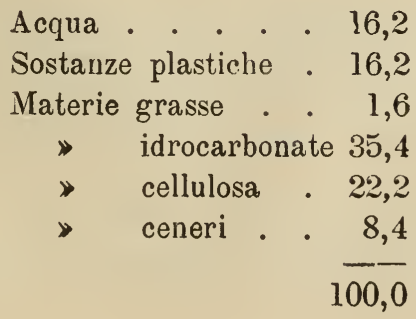


Il professore Tito Pasqui e professore Alessandro Pasqualini nel pregievolissimo loro studio sulle piante foraggiere in generale, stabiliscono per il trifoglio verde e per quello ridotto in fieno lo specchietto analitico seguente:

\begin{tabular}{|c|c|c|c|c|c|c|}
\hline & & & & & Verde & Asciutto \\
\hline Acqua . & - & & - & . & 67,040 & 16,520 \\
\hline Materie & grasse & . & . & • & 0,226 & 3,104 \\
\hline » & proteiche & . & . & . & 3,587 & 12,456 \\
\hline 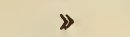 & idrocarbonat & & - & . & 20,888 & 35,440 \\
\hline Cellulosa & . & • & . & . & 4,430 & 18,382 \\
\hline Ceneri & . & . & . & . & 3,829 & 13,300 \\
\hline
\end{tabular}

Parti 100 ceneri della pianta verde

Ossido ferrico . . . 3,512

\ calcico . . . 33,278

» magnesiaco - 0,948

Ossido potassico e sodico 33.035

Acido fosforico . . 3,510

\ solforico . . 6,753

Cloro . . . . . 0,525

Anidride silicica . . 18,419
Parti 100 ceneri della pianta secca

Ossido ferrico . . . 5,014

\ calcico . . . 30,644

» magnesiaco . 1,887

Ossido di potassio . 28,594

» di sodio . . 2.689

Anidride fosforica . 4,907

» solforica . . 6,283

Cloro . . . . . 1,779

Anidr. silicica e silicati 18,045

Perdite. . . . 0,158

I quadri qui in appresso riportati sono invece quelli che rappresentano la composizione chimica immediata di alcuni campioni di trifoglio coltivati in varie località del Novarese, e delle loro ceneri, secondo $\mathrm{i}$ dati ottenuti mediante accurata analisi chimica.

Ho poi creduto opportuno di separare e analizzare a parte le foglie e gli steli per dimostrare con la esperienza alla 
mano, quanto piủ sopra ho detto, che cioe i principi nutritivi si plastici che respiratori si trovano in Imaggior copia nelle parti fogliacee che negli steli. 
Composizione immediata delle

Trifoglio verde.

Acqua

Materie grasse (estratto etereo)

Materıe proteiche $(15,5 \%$ azoto $)$

Cellulosa

Sostanze idrocarbonate (zucchero, amido, ecc.)

Materie minerali (cenere)

N. 1

\section{Trifoglio asciutto.}

Acqua igroscopica

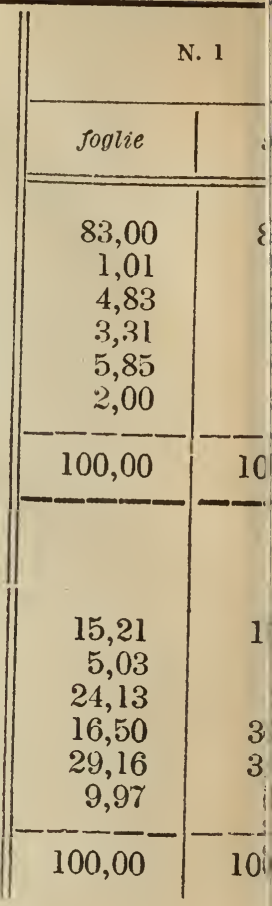

Compo

Silice e sabbia

Anidride carbonica

Cloro

Anidride solforica

Id. fosforica

Ossido di ferro

Id. di calcio

Id. di magnesio

Id. di potassio

Id. di sodio

\begin{tabular}{r|r}
4,20 & 3 \\
20,00 & 21 \\
1,15 & 1 \\
2,85 & 3 \\
7,88 & 6 \\
1,17 & 1 \\
20,55 & 21 \\
6,20 & 5 \\
28,70 & 25 \\
6,01 & 8 \\
\hline 98,71 & -2
\end{tabular}


Composizione immediata delle

Trifoglio verde.

Acqua

Materie grasse (estratto etereo)

Sostanze proteiche (15,5\% azoto) .

Cellulosa

Materie idrocarbonate (zucchero, amido, ecc.)

Materie minerali (cenere)

\section{Trifoglio asciutto.}

Acqua igroscopica

Materia grassa (estratto etereo) .

Sostanze proteiche $(15,5 \%$ azoto $)$. . . . Cellulosa

Materie idrocarbonate (zucchero, amido, ecc.) Materie saline (cenere)

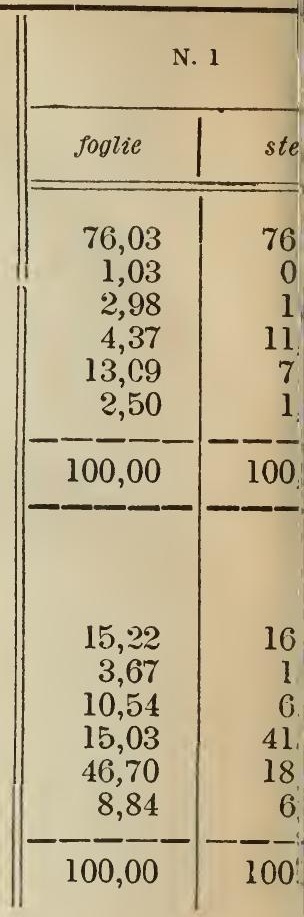

Compos

Sabbia e silice Anidride carbonica Cloro

Anidride solforica

Id. fosforica

Ossido di ferro

Id. di calce

Id. di magnesia

Id. di potassio

Id. di sodio

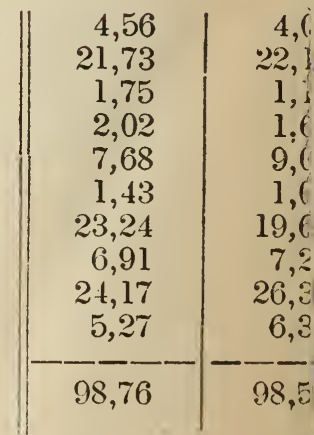


68

Basta ora gettare lo sguardo sopra questi quadri per convincersi della bontá come foraggio, dei nostri trifogli. In tutti i campioni infatti abbondano i principi grassi e proteici, non mancano nè scarseggiano le sostanze ternarie o respiratorie, e a celiulosa pure si trova in giusta proporzione. 



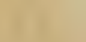




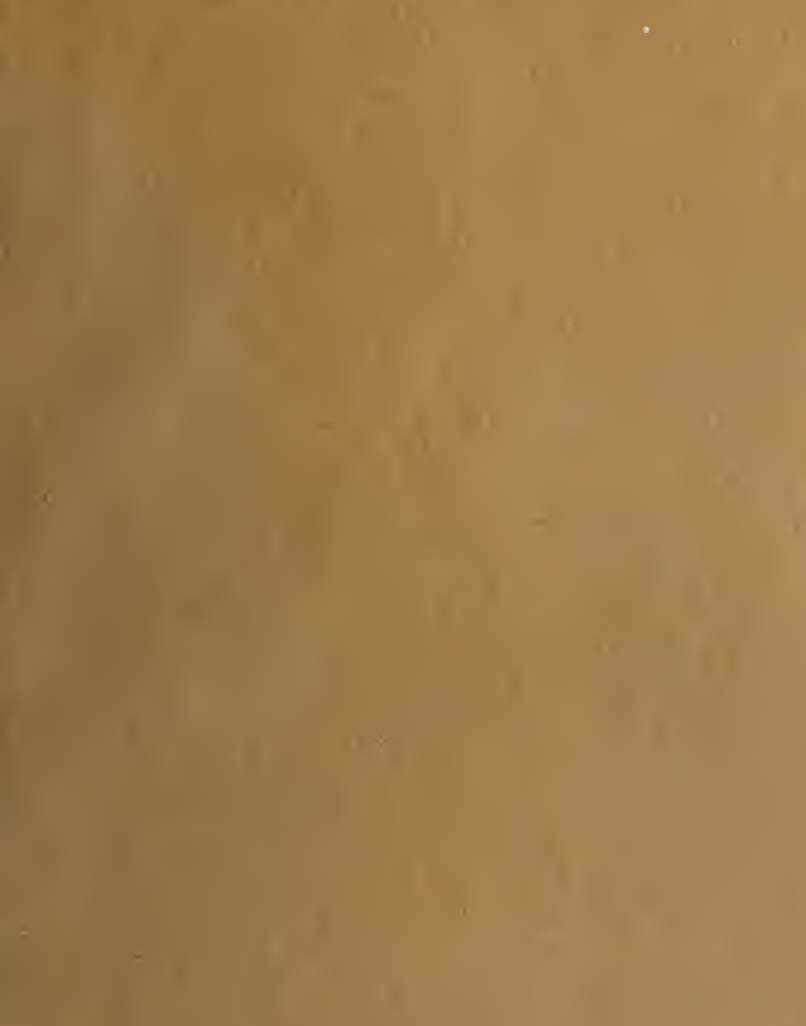

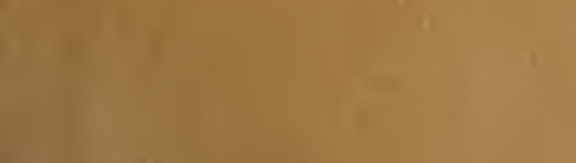

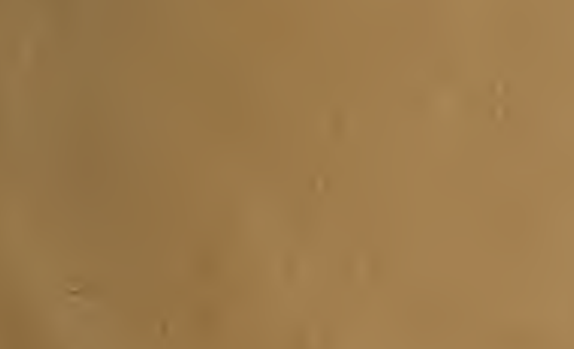

1
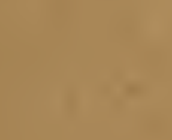

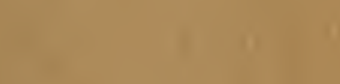
i

$x$

and a

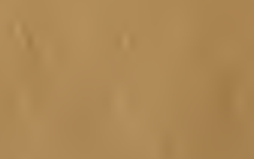
4 I) 

UNIVERSITY OF ILLINOIS-URBANA

||||||||||||||||||||||||||||||||||||||||||||||||||||||||||||

|||||||||||||||||||||||||||||||||||||||||| |||||||||||||||||||| 30112077123609 